FR-PHENO-2013-017

KA-TP-40-2013

PSI-PR-13-16

SFB/CPP-13-112

\title{
NMSSMCALC: \\ A Program Package for the Calculation of Loop-Corrected Higgs Boson Masses and Decay Widths in the (Complex) NMSSM
}

\author{
J. Baglio ${ }^{1}$, R. Gröber ${ }^{1}$, M. Mühlleitner ${ }^{1}$, D.T. Nhung ${ }^{1}$, H. Rzehak ${ }^{2}$, M. Spira ${ }^{3}$, \\ J. Streicher ${ }^{1}$ and K. Walz ${ }^{1}$ \\ ${ }^{1}$ Institut für Theoretische Physik, KIT, D-76128 Karlsruhe, Germany. \\ ${ }^{2}$ Albert-Ludwigs-Universität Freiburg, Physikalisches Institut, D-79104 Freiburg, Germany. \\ ${ }^{3}$ Paul Scherrer Institut, CH-5232 Villigen PSI, Switzerland.
}

\begin{abstract}
We present the program package NMSSMCALC for the calculation of the loop-corrected NMSSM Higgs boson masses and decay widths in the CP-conserving and CP-violating NMSSM. The full one-loop corrections to the Higgs boson masses are evaluated in a mixed renormalisation scheme of on-shell and $\overline{\mathrm{DR}}$ conditions. The Higgs decay widths include the dominant higher order QCD corrections, and the decays into bottom quarks, strange quarks and $\tau$ leptons are supplemented by higher order SUSY corrections through effective couplings. All relevant off-shell decays into two massive gauge bosons, gauge and Higgs boson and Higgs pair final states as well as into heavy quark pairs are computed. The input and output files feature the SUSY Les Houches Accord so that the program can easily be linked with existing computer tools.
\end{abstract}




\section{Introduction}

The announcement of the discovery of a new particle with a mass of about $126 \mathrm{GeV}$ by the LHC experiments ATLAS and CMS [1, 2] has marked a milestone in the history of particle physics. With the growing amount of data the experiments have started investigating the properties of this particle, i.e. its couplings to other Standard Model (SM) particles as well as its spin and parity quantum numbers. The results of these measurements strongly suggest the particle to be the Higgs boson, the particle predicted by the Higgs mechanism [3, 4, which allows to introduce particle masses without violating the gauge symmetries of the SM. While the data is compatible with a SM-like Higgs state it leaves room for interpretations within Higgs sectors of theories beyond the SM (BSM), among which supersymmetric (SUSY) extensions have been extensively studied. The correct interpretation of the data and the proper distinction between different models require, on the theoretical side, precise predictions for the masses and couplings of the investigated models as well as for the Higgs production cross sections and branching ratios, taking into account higher order corrections. The implementation of these calculations in public computer tools allows to test various models at the highest possible precision.

The Next-to-Minimal Supersymmetric Extension of the SM (NMSSM) [5] 7] with two complex Higgs doublets and one complex singlet field features after electroweak symmetry breaking (EWSB) seven Higgs bosons. In the CP-conserving case, these are three neutral CP-even, two neutral $\mathrm{CP}$-odd and two charged Higgs bosons. Allowing for $\mathrm{CP}$ violation the five neutral Higgs mass eigenstates, being mixtures of $\mathrm{CP}$-even and $\mathrm{CP}$-odd components already at tree level, do not carry definite $\mathrm{CP}$ quantum numbers anymore. Besides the benefits of supersymmetric theories in general the NMSSM is an attractive BSM extension as it solves the $\mu$ problem of the Minimal Supersymmetric Extension of the SM (MSSM). The $\mu$-parameter of the Higgs potential is generated dynamically through the singlet field acquiring a vacuum expectation value (VEV) so that it is naturally of the order of the scale of EWSB. Furthermore, with an additional tree-level contribution to the lighter MSSM-like Higgs boson mass, proportional to the doublet-singlet coupling $\lambda$, it requires smaller radiative corrections to achieve a mass of $126 \mathrm{GeV}$, and hence smaller stop masses and/or mixing, implying less fine-tuning [8]. The extended Higgs sector finally, entails a plethora of interesting phenomenological implications, like Higgs-to-Higgs decays, suppressed or enhanced branching ratios compared to the SM or a Higgs signal which is built up by two resonances close in mass around $126 \mathrm{GeV}$, to cite only a few of them. This makes clear that precise predictions for the masses of the NMSSM Higgs states and for their production and decay processes including higher order corrections are needed to interpret the experimental data reliably.

We present the program package NMSSMCALC1 1 for the calculation of loop-corrected NMSSM Higgs boson masses as well as of the decay widths and branching ratios, both for the CP-conserving and the CP-violating case. The package includes

- The computation of the full one-loop corrections to the NMSSM Higgs boson masses in a mixed renormalisation scheme of on-shell and $\overline{\mathrm{DR}}$ conditions both in the CP-conserving [10] and in the CP-violating NMSSM [11] 2

- The calculation of the Higgs decay widths and branching ratios in the CP-conserving and CP-violating implementations of the NMSSM. This part of the program package is based on an extension of the Fortran code HDECAY [22]. The decay widths include the dominant

\footnotetext{
${ }^{1} \mathrm{~A}$ first version of the program has been presented in 9 .

${ }^{2}$ For further higher order calculations to the real NMSSM Higgs boson masses, see [12 15], and for the complex case see Refs. [16-20]. Corrections to the trilinear Higgs self-couplings have been provided in [21.
} 
higher order QCD corrections. For the neutral Higgs boson decays into a bottom quark pair furthermore the higher order SUSY-QCD and the approximate SUSY-electroweak (elw) corrections up to one-loop accuracy have been implemented. They have been obtained by adapting the existing results for the MSSM 23 28, to the NMSSM case. The decays into a strange quark pair include the dominant resummed SUSY-QCD corrections and the one into a $\tau$ pair the dominant resummed SUSY-elw corrections, again by adapting them from the MSSM to the NMSSM Higgs bosons. Analogously for the charged Higgs boson the higher order SUSY corrections have been implemented for the decays into fermion pairs. In the real NMSSM, the decays into stop and sbottom pairs, respectively, contain the SUSY-QCD corrections.

- The inclusion of all relevant off-shell decays into two massive gauge boson final states, into gauge and Higgs boson final states, into Higgs pairs as well as into heavy quark pairs.

- The input and output files feature the SUSY Les Houches Accord (SLHA) [29, 30] so that the program package can easily be linked with other existing computer tools.

In general, the electroweak corrections going beyond the approximate SUSY-elw contribution, have not been taken into account in the calculation of the decay widths and branching ratios. They are available for some MSSM decays and could in principle be extended to the NMSSM case. Due to the additional NMSSM singlet field, this requires, however, further calculations, which are beyond the scope of the present implementation. We leave this for future work.

The outline of the draft is as follows. After introducing the NMSSM Lagrangian in section 2 we present in section 3 the main features of the calculation of the one-loop corrections to the NMSSM Higgs boson masses in the CP-conserving NMSSM, and in section 4 in the CP-violating NMSSM. Section 5 gives an overview of the implemented decays and their higher order corrections. In particular we present in subsection 5.3 the higher order SUSY-QCD and SUSY-elw corrections to the NMSSM Higgs boson decays into quark pairs. In section 6 the program package is described with its main routines, input and output files, and how to run the program. Section 7 deals with various issues related to the SUSY Les Houches Accord with respect to our program package. In section 8 we summarise and give an outlook on future developments. The program package can be found at the url: http://www.itp.kit.edu/ maggie/NMSSMCALC/.

\section{The NMSSM Lagrangian}

With respect to the MSSM Lagrangian, the NMSSM differs by the superpotential and the soft SUSY breaking part. Denoting the Higgs doublet superfields, which couple to the up- and downtype quarks, by $\hat{H}_{u}$ and $\hat{H}_{d}$, respectively, and the singlet superfield by $\hat{S}$, the scale invariant superpotential reads

$$
W_{N M S S M}=W_{M S S M}-\epsilon_{i j} \lambda \hat{S} \hat{H}_{d}^{i} \hat{H}_{u}^{j}+\frac{1}{3} \kappa \hat{S}^{3},
$$

with the $S U(2)_{L}$ indices $i, j=1,2$ and the totally antisymmetric tensor $\epsilon_{12}=1$. While the dimensionless parameters $\lambda$ and $\kappa$ can be complex in general, in case of CP-conservation they are taken to be real. In terms of the quark and lepton superfields and their charge conjugates, indicated by the superscript $c, \hat{Q}, \hat{U}^{c}, \hat{D}^{c}, \hat{L}, \hat{E}^{c}$, the MSSM superpotential $W_{M S S M}$ is given by

$$
W_{M S S M}=\epsilon_{i j}\left[y_{e} \hat{H}_{d}^{i} \hat{L}^{j} \hat{E}^{c}+y_{d} \hat{H}_{d}^{i} \hat{Q}^{j} \hat{D}^{c}-y_{u} \hat{H}_{u}^{i} \hat{Q}^{j} \hat{U}^{c}\right]
$$


where the colour and generation indices have been suppressed. The Yukawa couplings $y_{d}, y_{u}$ and $y_{e}$ in the MSSM superpotential Eq. (2) are in general complex. Their phases can, however, be reabsorbed, by redefining the quark fields, if generation mixing is neglected as it is done in our approach for the calculation of the Higgs masses. The MSSM $\mu$-term as well as the tadpole and bilinear terms of $\hat{S}$ are assumed to be zero. Denoting the Higgs doublet and singlet component fields by $H_{u}, H_{d}$ and $S$, the soft SUSY breaking NMSSM Lagrangian is given by

$$
\mathcal{L}_{\text {soft }}=\mathcal{L}_{\text {soft }, M S S M}-m_{S}^{2}|S|^{2}+\left(\epsilon_{i j} \lambda A_{\lambda} S H_{d}^{i} H_{u}^{j}-\frac{1}{3} \kappa A_{\kappa} S^{3}+\text { h.c. }\right),
$$

with the soft SUSY breaking MSSM Lagrangian

$$
\begin{aligned}
\mathcal{L}_{\text {soft }, M S S M}= & -m_{H_{d}}^{2} H_{d}^{\dagger} H_{d}-m_{H_{u}}^{2} H_{u}^{\dagger} H_{u}-m_{Q}^{2} \tilde{Q}^{\dagger} \tilde{Q}-m_{L}^{2} \tilde{L}^{\dagger} \tilde{L}-m_{U}^{2} \tilde{u}_{R}^{*} \tilde{u}_{R}-m_{D}^{2} \tilde{d}_{R}^{*} \tilde{d}_{R} \\
& -m_{E}^{2} \tilde{e}_{R}^{*} \tilde{e}_{R}-\left(\epsilon_{i j}\left[y_{e} A_{e} H_{d}^{i} \tilde{L}^{j} \tilde{e}_{R}^{*}+y_{d} A_{d} H_{d}^{i} \tilde{Q}^{j} \tilde{d}_{R}^{*}-y_{u} A_{u} H_{u}^{i} \tilde{Q}^{j} \tilde{u}_{R}^{*}\right]+\text { h.c. }\right) \\
& -\frac{1}{2}\left(M_{1} \tilde{B} \tilde{B}+M_{2} \tilde{W}_{k} \tilde{W}_{k}+M_{3} \tilde{G} \tilde{G}+\text { h.c. }\right) .
\end{aligned}
$$

Here $\tilde{B}, \tilde{W}_{k}(k=1,2,3)$ and $\tilde{G}$ are the gaugino fields, and $\tilde{Q}=\left(\tilde{u}_{L}, \tilde{d}_{L}\right)^{T}, \tilde{L}=\left(\tilde{\nu}_{L}, \tilde{e}_{L}\right)^{T}$, where the tilde denotes the scalar components of the corresponding quark and lepton superfields. In the soft SUSY breaking NMSSM Lagrangian Eq. (3) the soft SUSY breaking mass parameters $m_{X}^{2}$ of the scalar fields $X=S, H_{d}, H_{u}, Q, U, D, L, E$ are real. The soft SUSY breaking trilinear couplings $A_{x}(x=\lambda, \kappa, d, u, e)$ and the gaugino mass parameters $M_{1}, M_{2}$ and $M_{3}$, however, are complex in general but real in the CP-conserving case. We furthermore neglect squark and slepton mixing between the generations and set soft SUSY breaking terms linear and quadratic in the singlet field $S$ to zero.

\section{Loop-corrected Higgs boson masses in the real NMSSM}

In this section we summarise the main features of the calculation of the one-loop corrections to the NMSSM Higgs boson masses in the CP-conserving NMSSM. For details we refer the reader to Ref. [10].

\subsection{The CP-conserving NMSSM Higgs sector}

The Higgs mass matrix is derived from the NMSSM Higgs potential, which is obtained from the superpotential, the soft SUSY breaking terms and from the $D$-term contributions. The Higgs doublet and singlet fields entering the Higgs potential acquire non-vanishing vacuum expectation values (VEVs) after electroweak symmetry breaking. After expansion of the Higgs fields about their VEVs $v_{u}, v_{d}$ and $v_{s}$, chosen to be real and positive,

$$
H_{d}=\left(\begin{array}{c}
\left(v_{d}+h_{d}+i a_{d}\right) / \sqrt{2} \\
h_{d}^{-}
\end{array}\right), \quad H_{u}=\left(\begin{array}{c}
h_{u}^{+} \\
\left(v_{u}+h_{u}+i a_{u}\right) / \sqrt{2}
\end{array}\right), \quad S=\frac{v_{s}+h_{s}+i a_{s}}{\sqrt{2}},
$$

the $3 \times 3$ Higgs mass matrices squared for the CP-even and CP-odd component Higgs fields, $M_{S}^{2}$ and $M_{A}^{2}$, respectively, can be derived from the tree-level scalar potential. Explicit expressions can be found in [10. The squared mass matrix $M_{S}^{2}$ is diagonalised through a rotation $\mathcal{R}^{S}$, yielding the CP-even mass eigenstates $H_{i}(i=1,2,3)$,

$$
\left(H_{1}, H_{2}, H_{3}\right)^{T}=\mathcal{R}^{S}\left(h_{d}, h_{u}, h_{s}\right)^{T}, \quad \operatorname{diag}\left(\left(M_{H_{1}}^{(0)}\right)^{2},\left(M_{H_{2}}^{(0)}\right)^{2},\left(M_{H_{3}}^{(0)}\right)^{2}\right)=\mathcal{R}^{S} M_{S}^{2}\left(\mathcal{R}^{S}\right)^{T} .
$$


The mass eigenstates are ordered by ascending mass, $M_{H_{1}}^{(0)} \leq M_{H_{2}}^{(0)} \leq M_{H_{3}}^{(0)}$, where the superscript (0) indicates the tree-level mass values. In the CP-odd Higgs sector a first rotation $\mathcal{R}^{G}$ is applied to separate the massless Goldstone boson $G$, followed by a rotation $\mathcal{R}^{P}$ to obtain the mass eigenstates $A_{i} \equiv A_{1}, A_{2}, G(i=1,2,3), c f .[10]$,

$$
\left(A_{1}, A_{2}, G\right)^{T}=\mathcal{R}^{P} \mathcal{R}^{G}\left(a_{d}, a_{u}, a_{s}\right)^{T}, \quad \operatorname{diag}\left(\left(M_{A_{1}}^{(0)}\right)^{2},\left(M_{A_{2}}^{(0)}\right)^{2}, 0\right)=\mathcal{R}^{P} \mathcal{R}^{G} M_{A}^{2}\left(\mathcal{R}^{P} \mathcal{R}^{G}\right)^{T} .
$$

At tree-level the CP-conserving NMSSM Higgs potential depends on 12 independent parameters, which are the $U(1)$ and $S U(2)$ gauge couplings $g_{1}$ and $g_{2}$, the three VEVs, the soft SUSY breaking mass parameters of the doublet and the singlet Higgs fields and the NMSSM specific parameters and soft SUSY breaking couplings,

$$
g_{1}, g_{2}, v_{d}, v_{u}, v_{s}, m_{H_{d}}^{2}, m_{H_{u}}^{2}, m_{S}^{2}, \lambda, \kappa, A_{\lambda}, A_{\kappa} .
$$

Some of these parameters are replaced in order to obtain a more transparent physical interpretation. The minimisation conditions of the Higgs potential $V$ require the terms linear in the Higgs fields to vanish in the vacuum. Hence, at tree-level, for the scalar fields,

$$
\left.t_{\phi} \equiv \frac{\partial V}{\partial \phi}\right|_{\text {Min }} \stackrel{!}{=} 0 \quad \text { for } \quad \phi=h_{d}, h_{u}, h_{s}
$$

These conditions are used to trade $m_{H_{d}}^{2}, m_{H_{u}}^{2}$ and $m_{S}^{2}$ for the tadpole parameters $t_{h_{d}}, t_{h_{u}}$ and $t_{h_{s}}$. The soft SUSY breaking coupling $A_{\lambda}$ can optionally be replaced by the charged Higgs boson mass $M_{H^{ \pm}}$, and the parameters $g_{1}, g_{2}, v_{u}, v_{d}$ are substituted by the gauge boson masses $M_{W}$ and $M_{Z}$, the electric charge $e$ and $\tan \beta$, where $\tan \beta$ is the ratio of the two vacuum expectation values of the doublet fields,

$$
\tan \beta=\frac{v_{u}}{v_{d}} .
$$

We hence have two possible new parameter sets to work with

$$
\begin{aligned}
\text { 1st parameter set: } & M_{Z}, M_{W}, M_{H^{ \pm}}, t_{h_{d}}, t_{h_{u}}, t_{h_{s}}, e, \tan \beta, \lambda, v_{s}, \kappa, A_{\kappa} ; \\
\text { 2nd parameter set: } & M_{Z}, M_{W}, t_{h_{d}}, t_{h_{u}}, t_{h_{s}}, e, \tan \beta, \lambda, A_{\lambda}, v_{s}, \kappa, A_{\kappa} .
\end{aligned}
$$

Note that the first one is the one we chose in our previous work [10], whereas the second one provides an additional option.

\subsection{One-loop corrected Higgs boson masses in the real NMSSM}

The Higgs self-energies calculated for the determination of the loop-corrected Higgs boson masses develop ultraviolet (UV) divergencies. The renormalisation of the parameters entering the loop calculation renders the result finite. The two renormalisation schemes which we apply to the two parameter sets are a mixture of on-shell and $\overline{\mathrm{DR}}$ renormalisation conditions as follows:

$$
\begin{aligned}
\text { 1st renormalisation scheme: } & \underbrace{M_{Z}, M_{W}, M_{H^{ \pm}}, t_{h_{d}}, t_{h_{u}}, t_{h_{s}}, e}_{\text {on-shell scheme }}, \underbrace{\tan \beta, \lambda, v_{s}, \kappa, A_{\kappa}}_{\overline{\mathrm{DR}} \text { scheme }} ; \\
\text { 2nd renormalisation scheme: } & \underbrace{M_{Z}, M_{W}, t_{h_{d}}, t_{h_{u}}, t_{h_{s}}, e}_{\text {on-shell scheme }}, \underbrace{\tan \beta, \lambda, A_{\lambda}, v_{s}, \kappa, A_{\kappa}}_{\overline{\mathrm{DR}} \text { scheme }} .
\end{aligned}
$$

The specific form of the counterterms for the first scheme can be found in [10]. The only modification in the second scheme is that the counterterm to $A_{\lambda}$ is determined from the charged Higgs sector 
via a $\overline{\mathrm{DR}}$ condition. In both schemes the chargino sector is exploited in the derivation of the counterterm related to $v_{s}$, and the neutralino sector in the derivation of the counterterm for $\kappa$. The field renormalisation of the Higgs boson doublet and singlet fields are defined via $\overline{\mathrm{DR}}$ conditions. External self-energy contributions are properly taken into account, cf. [10,31 for details.

The parameters given in the sets Eqs. (11) and (12) are the ones which we use in the calculation of the mass corrections and on which we apply our renormalisation conditions. However, they are not the input parameters, which are to be provided by the user. These are the SM inputs as defined in the SLHA 3 , the value for $\tan \beta$, the soft SUSY breaking gaugino and squark mass parameters, the soft SUSY breaking trilinear couplings and the NMSSM specific parameters $\lambda, \kappa, A_{\lambda}, A_{\kappa}$ and $\mu_{\text {eff }}$. The latter is generated dynamically when the Higgs singlet field $S$ acquires its vacuum expectation value $\langle S\rangle$,

$$
\mu_{\mathrm{eff}}=\lambda\langle S\rangle \equiv \lambda \frac{v_{s}}{\sqrt{2}} .
$$

Alternatively, for $A_{\lambda}$ the charged Higgs boson mass $M_{H^{ \pm}}$can be provided.

The one-loop corrected scalar Higgs boson masses squared are determined numerically and given by the real parts of the zeroes of the determinant of the two-point vertex functions $\hat{\Gamma}^{S}$,

$$
\begin{aligned}
& \hat{\Gamma}^{S}\left(k^{2}\right)= \\
& \quad i\left(\begin{array}{ccc}
k^{2}-\left(M_{H_{1}}^{(0)}\right)^{2}+\hat{\Sigma}_{H_{1} H_{1}}\left(k^{2}\right) & \hat{\Sigma}_{H_{1} H_{2}}\left(k^{2}\right) & \hat{\Sigma}_{H_{1} H_{3}}\left(k^{2}\right) \\
\hat{\Sigma}_{H_{2} H_{1}}\left(k^{2}\right) & k^{2}-\left(M_{H_{2}}^{(0)}\right)^{2}+\hat{\Sigma}_{H_{2} H_{2}}\left(k^{2}\right) & \hat{\Sigma}_{H_{2} H_{3}}\left(k^{2}\right) \\
\hat{\Sigma}_{H_{3} H_{1}}\left(k^{2}\right) & \hat{\Sigma}_{H_{3} H_{2}}\left(k^{2}\right) & k^{2}-\left(M_{H_{3}}^{(0)}\right)^{2}+\hat{\Sigma}_{H_{3} H_{3}}\left(k^{2}\right)
\end{array}\right)
\end{aligned}
$$

while the pseudoscalar masses squared are extracted from $\hat{\Gamma}^{P}$,

$$
\hat{\Gamma}^{P}\left(k^{2}\right)=i\left(\begin{array}{cc}
k^{2}-\left(M_{A_{1}}^{(0)}\right)^{2}+\hat{\Sigma}_{A_{1} A_{1}}\left(k^{2}\right) & \hat{\Sigma}_{A_{1} A_{2}}\left(k^{2}\right) \\
\hat{\Sigma}_{A_{2} A_{1}}\left(k^{2}\right) & k^{2}-\left(M_{A_{2}}^{(0)}\right)^{2}+\hat{\Sigma}_{A_{2} A_{2}}\left(k^{2}\right)
\end{array}\right) .
$$

Note that in the pseudoscalar sector no mixing with the would-be Goldstone bosons is taken into account, since we checked that the effect is numerically negligible. Also no mixing with the longitudinal component of the $Z$ boson is taken into account, as it has been shown in the MSSM [32] that it is sufficient to include the mixing with the would-be Goldstone boson. The unrenormalised self-energies and tadpole conditions appearing implicitly in the renormalised self-energies $\hat{\Sigma}$ are evaluated at one-loop order. The mass eigenvalues are obtained in an iterative procedure keeping the full dependence on the external momentum squared $k^{2}$ in the renormalised self-energies. The Higgs mixing matrix elements on the other hand are obtained by setting $k^{2}=0$, corresponding to a proper definition of the effective mixing matrix elements. This yields a unitary mixing matrix 4 The differences between this approach and the one with non-vanishing $k^{2}$ have been found to be small in the investigated parameter sets.

In the first renormalisation scheme the mass of the charged Higgs boson is renormalised on-shell, and therefore constitutes the physical value even at one-loop order. If, however, the second scheme

\footnotetext{
${ }^{3}$ They are given by the inverse electromagnetic coupling and the strong coupling at the $Z$ pole in the $\overline{\mathrm{MS}}$ scheme with five active flavours, the Fermi constant, the $Z$ pole mass, the running mass of the $b$-quark in the $\overline{M S}$ scheme and the top and tau pole masses. Additionally the $W$ boson pole mass has to be given. This value is not included in the original SLHA SM inputs, but needed in the calculation of the loop-corrected Higgs boson masses and of the decay widths.

${ }^{4}$ For non-vanishing $k^{2}$ the mixing matrix is not unitary.
} 
is applied the pole mass of the charged Higgs boson at one-loop level, $M_{H^{ \pm}}^{1 \text { loop }}$, is determined by iteratively solving the equation

$$
\left(M_{H^{ \pm}}^{\text {1loop }}\right)^{2}=\left(M_{H^{ \pm}}^{\text {tree }}\right)^{2}-\hat{\Sigma}_{H^{ \pm} H^{ \pm}}\left(\left(M_{H^{ \pm}}^{\text {1loop }}\right)^{2}\right),
$$

with $\hat{\Sigma}_{H^{ \pm} H^{ \pm}}$denoting the renormalised self-energy of the charged Higgs boson which is given by

$$
\hat{\Sigma}_{H^{ \pm} H^{ \pm}}=\Sigma_{H^{ \pm} H^{ \pm}}\left(k^{2}\right)+\left(k^{2}-M_{H^{ \pm}}^{2}\right)\left(s_{\beta}^{2} \delta Z_{H_{d}}+c_{\beta}^{2} \delta Z_{H_{u}}\right)-\delta M_{H^{ \pm}}^{2},
$$

where the counterterm $\delta M_{H^{ \pm}}^{2}$ is a function of the counterterms of the second renormalisation scheme and the wavefunction renormalisation factors are renormalised in the $\overline{\mathrm{DR}}$ scheme. Again, external self-energy contributions are properly taken into account [10]. The mixing of the charged Higgs boson with the charged would-be Goldstone boson is neglected.

Throughout the calculation of the one-loop masses the running $\overline{\mathrm{DR}}$ top and bottom quark masses are used.

\section{Loop-corrected Higgs boson masses in the complex NMSSM}

The calculation of the Higgs boson masses in the CP-violating NMSSM is a generalization of the real case described in section 3.2. Choosing vanishing phases and imaginary parts, respectively, yields the same result as before. Hence, in this section we restrict ourselves to summarising the differences of the CP-violating NMSSM with respect to the CP-conserving NMSSM described in the previous section. For further details, we refer the reader to Ref. [11].

\subsection{The CP-violating NMSSM Higgs sector}

In addition to the complex parameters present in the MSSM, there are four more parameters in the NMSSM, that can become complex. These are the couplings $\lambda, \kappa$ and the soft SUSY breaking trilinear couplings $A_{\lambda}$ and $A_{\kappa}$. The complex parameters originating from the MSSM part are the soft SUSY breaking trilinear couplings $A_{u}, A_{d}, A_{e}$ of the up-type, down-type and charged leptontype sfermions, respectively, as well as the soft SUSY breaking mass parameters of the gauginos $M_{1}, M_{2}$ and $M_{3}$. $R$-symmetry can be exploited to choose either $M_{1}$ or $M_{2}$ to be real. We do not make use of this symmetry, however, in order to keep the relations as general as possible. The kinetic and gauge interaction parts of the NMSSM Lagrangian contain no complex parameters.

In the expansion of the Higgs boson fields about the VEVs two further phases, $\varphi_{u}$ and $\varphi_{s}$, appear,

$$
H_{d}=\left(\begin{array}{c}
\frac{1}{\sqrt{2}}\left(v_{d}+h_{d}+i a_{d}\right) \\
h_{d}^{-}
\end{array}\right), \quad H_{u}=e^{i \varphi_{u}}\left(\begin{array}{c}
h_{u}^{+} \\
\frac{1}{\sqrt{2}}\left(v_{u}+h_{u}+i a_{u}\right)
\end{array}\right), \quad S=\frac{e^{i \varphi_{s}}}{\sqrt{2}}\left(v_{s}+h_{s}+i a_{s}\right) .
$$

They describe the phase differences between the three VEVs $\left\langle H_{d}^{0}\right\rangle,\left\langle H_{u}^{0}\right\rangle$ and $\langle S\rangle$. For phase values $\varphi_{u}=\varphi_{s}=n \pi, n \in \mathbb{N}$, the fields $h_{i}$ and $a_{i}(i=d, u, s)$ are the pure CP-even and CP-odd parts of the neutral entries of $H_{u}, H_{d}$ and $S$. We exploit the freedom in the phase choice of the Yukawa couplings to set $\varphi_{y_{u}}=-\varphi_{u}$ and assume the down-type and charged lepton-type Yukawa couplings to be real. In this way the quark and lepton mass terms yield real masses without any further phase transformation of the corresponding fields.

After the expansion about the VEVs the Higgs boson mass matrix $M_{\phi \phi}$ can be read off from the terms in the Higgs potential which are bilinear in the neutral Higgs boson fields. CP-violation 
introduces a mixing between CP-even and CP-odd component fields, so that this matrix is a $6 \times 6$ matrix in the basis $\phi=\left(h_{d}, h_{u}, h_{s}, a_{d}, a_{u}, a_{s}\right)^{T}$, which can be expressed in terms of three $3 \times 3$ matrices $M_{h h}, M_{a a}$ and $M_{h a}$,

$$
M_{\phi \phi}=\left(\begin{array}{ll}
M_{h h} & M_{h a} \\
M_{h a}^{T} & M_{a a}
\end{array}\right)
$$

where $M_{h h}$ and $M_{a a}$ are symmetric matrices, describing the mixing among the CP-even components of the Higgs doublet and singlet fields and among the CP-odd components, respectively. In case of CP-conservation the matrix $M_{h a}$, which mixes CP-even and CP-odd components, vanishes. Note that due to the application of the minimisation conditions of the Higgs potential $V$,

$$
\left.t_{\phi} \equiv \frac{\partial V}{\partial \phi}\right|_{\mathrm{Min}} \stackrel{!}{=} 0 \quad \text { for } \quad \phi=h_{d}, h_{u}, h_{s}, a_{d}, a_{u}, a_{s}
$$

in the tree-level Higgs sector only one linearly independent phase combination $\varphi_{y}$ appears after applying the tadpole conditions for $\phi=a_{d}$ and $a_{s} 5$,

$$
\varphi_{y}=\varphi_{\kappa}-\varphi_{\lambda}+2 \varphi_{s}-\varphi_{u}
$$

Hence the CP mixing due to $M_{h a}$ is governed by $\sin \varphi_{y}$ at tree-level.

The transformation into mass eigenstates is performed by subsequently applying the $6 \times 6$ rotation matrix $\mathcal{R}^{G}$ to separate the would-be Goldstone boson field and then the matrix $\mathcal{R}$ to rotate to the mass eigenstates $H_{i}(i=1, \ldots, 5)$, yielding a diagonal mass matrix squared,

$$
\begin{aligned}
\left(H_{1}, H_{2}, H_{3}, H_{4}, H_{5}, G\right)^{T} & =\mathcal{R}^{D}\left(h_{d}, h_{u}, h_{s}, a_{d}, a_{u}, a_{s}\right)^{T} \\
\operatorname{diag}\left(\left(M_{H_{1}}^{(0)}\right)^{2}, \ldots,\left(M_{H_{5}}^{(0)}\right)^{2}, 0\right) & =\mathcal{R}^{D} M_{\phi \phi}\left(\mathcal{R}^{D}\right)^{T},
\end{aligned}
$$

with $\mathcal{R}^{D} \equiv \mathcal{R} \mathcal{R}^{G}$ and the superscript (0) indicating tree-level masses.

At tree-level the parameters which enter the Higgs potential of the CP-violating NMSSM are

$$
m_{H_{d}}^{2}, m_{H_{u}}^{2}, m_{S}^{2}, g_{1}, g_{2}, v_{u}, v_{d}, v_{s}, \varphi_{s}, \varphi_{u}, \operatorname{Re} \lambda, \operatorname{Im} \lambda, \operatorname{Re} A_{\lambda}, \operatorname{Im} A_{\lambda}, \operatorname{Re} \kappa, \operatorname{Im} \kappa, \operatorname{Re} A_{\kappa}, \operatorname{Im} A_{\kappa} .
$$

Again we trade some of the parameters for more physical ones and use the following two parameter sets

$$
\begin{aligned}
& \underbrace{t_{h_{d}}, t_{h_{u}}, t_{h_{s}}, t_{a_{d}}, t_{a_{s}}, M_{H^{ \pm}}^{2}, M_{W}^{2}, M_{Z}^{2}, e}_{\text {on-shell }}, \underbrace{\tan \beta, v_{s}, \varphi_{s}, \varphi_{u}, \operatorname{Re} \lambda, \operatorname{Im} \lambda, \operatorname{Re} \kappa, \operatorname{Im} \kappa, \operatorname{Re} A_{\kappa}}_{\overline{\mathrm{DR}}} ; \\
& \underbrace{t_{h_{d}}, t_{h_{u}}, t_{h_{s}}, t_{a_{d}}, t_{a_{s}}, M_{W}^{2}, M_{Z}^{2}, e}_{\text {on-shell }}, \underbrace{\tan \beta, v_{s}, \varphi_{s}, \varphi_{u}, \operatorname{Re} \lambda, \operatorname{Im} \lambda, \operatorname{Re} A_{\lambda}, \operatorname{Re} \kappa, \operatorname{Im} \kappa, \operatorname{Re} A_{\kappa}}_{\overline{\mathrm{DR}}} \cdot
\end{aligned}
$$

The first part of parameters is defined via on-shell conditions and thus related to physical quantities. This also holds for the tadpole parameters, as their introduction is motivated by physical interpretation. The remaining parameters are interpreted as $\overline{\mathrm{DR}}$ parameters. Note that instead of splitting the complex parameters $\lambda, \kappa, A_{\lambda}$ and $A_{\kappa}$ into their absolute values and phases, as we did in our previous work [11, we split them into their real and imaginary parts. This allows us to

\footnotetext{
${ }^{5}$ The tadpole condition for $a_{u}$ does not lead to a new linearly independent condition. Note, that in the real case, the CP-odd tadpole conditions vanish and are thus automatically fulfilled.
} 
be in accordance with the SLHA conventions which require the real part of $A_{\lambda}$ and $A_{\kappa}$ to be $\overline{\mathrm{DR}}$ parameters. Expressing the imaginary parts of $A_{\lambda}$ and $A_{k}$ by the tadpole parameters $t_{a_{d}}$ and $t_{a_{s}}$ then leads to a slightly modified renormalisation scheme 6 . The two renormalisation schemes we provide based on the two parameter sets given above differ by either using the mass of the charged Higgs boson as an on-shell input or the real part of $A_{\lambda}$ as a $\overline{\mathrm{DR}}$ parameter.

Again the parameters given in the sets Eqs. (26) and (27) are the ones used in the calculation of the mass corrections and on which the renormalisation conditions are applied. The input parameters provided by the user, however, are the same as the ones for the real NMSSM, supplemented in the complex NMSSM by the imaginary parts of the soft SUSY breaking gaugino mass parameters, of the soft SUSY breaking trilinear couplings and of $\lambda, \kappa, \mu_{\text {eff }}$ as well as the phase $\varphi_{u}$, depending on the CP-violating scenario under investigation. The imaginary parts of $A_{\lambda}$ and $A_{\kappa}$ are already fixed by the tadpole conditions. Note that in the complex case the effective $\mu$-parameter $\mu_{\text {eff }}$ is defined as

$$
\mu_{\mathrm{eff}}=\lambda\langle S\rangle \equiv \lambda \frac{v_{s} e^{i \varphi_{s}}}{\sqrt{2}}
$$

\subsection{One-loop corrected NMSSM Higgs boson masses in the complex NMSSM}

The one-loop corrected neutral Higgs boson masses squared are determined numerically as the real parts of the zeroes of the determinant of the two-point vertex functions $\hat{\Gamma}$,

$$
\hat{\Gamma}\left(k^{2}\right)=i\left(\mathbb{1} \cdot k^{2}-\mathcal{M}^{1 \mathrm{l}}\right) \quad \text { with } \quad\left(\mathcal{M}^{1 \mathrm{l}}\right)_{i j}=\left(M_{H_{i}}^{(0)}\right)^{2} \delta_{i j}-\hat{\Sigma}_{i j}\left(k^{2}\right) \quad i, j=1, \ldots, 5,
$$

where the superscript 11 denotes the one-loop order. The specific form of the renormalised selfenergies $\hat{\Sigma}_{i j}$ in terms of the 1-loop self-energies, field renormalisation matrices and counterterms, to render the self-energies finite, can be found in [11. This reference includes the detailed description of the field renormalisation and of the renormalisation of the parameters given in Eq. (26). We make use of the chargino and neutralino sector in order to determine the counterterms of $v_{s}, \varphi_{s}, \lambda, \kappa$ and $\varphi_{u}$ as well as those of $M_{1}$ and $M_{2}$.

As in the CP-conserving case the one-loop Higgs masses are obtained iteratively by keeping the dependence on the external momentum squared in the renormalised self-energies. The mixing elements of the matrix performing the rotation from the interaction to the mass eigenstates at oneloop level are extracted for zero external momenta, i.e. the rotation matrix relating the tree-level to the one-loop mass eigenstates is defined as the matrix which diagonalises $\mathcal{M}^{11}$ after setting the momenta in $\mathcal{M}^{11}$ to zero. While this procedure does not retain the full momentum dependence it has the advantage of yielding a mixing matrix which is unitary. We have checked numerically that the difference with respect to the result keeping the full momentum dependence is negligible. Once again we use the running top and bottom quark masses, and the charged Higgs boson mass is obtained as before in the CP-conserving case.

\section{Decay Widths}

The spectrum file with the loop-corrected NMSSM Higgs masses and mixings and all SUSY particles with corresponding mixing angles is used in the routine which calculates the decay widths. The included decays are the NMSSM Higgs boson decays into SM and SUSY particle pairs as well as off-shell decays into 3- or 4-particle final states. They are described in more detail in the following.

\footnotetext{
${ }^{6}$ This change in the renormalisation scheme does not lead to any significant changes for the loop corrected mass values.
} 


\subsection{Decay Widths in the CP-conserving NMSSM}

Decays into quark pairs: The neutral Higgs decay widths into quark pairs receive QCD corrections which are available for the SM including the fully massive next-to-leading order (NLO) corrections near threshold [33] and massless $\mathcal{O}\left(\alpha_{s}^{4}\right)$ corrections far above threshold [34 36]. Furthermore large logarithms are resummed through the running of the quark masses and the strong coupling constant. The QCD corrections for the charged Higgs boson decay into a heavy quark pair have been given in [37. As these QCD corrections factorise with respect to the tree-level amplitude they can be taken over for the NMSSM Higgs decays and have been included in the decay code. In the decays into bottom quarks, apart from the QCD corrections, higher order SUSY corrections have been included by absorbing them into effective Yukawa couplings. They include the resummation of the dominant corrections for large values of $\tan \beta[23$ 25] and the SUSY-QCD corrections to the leading SUSY-QCD and top-induced SUSY-elw contributions [26 28]. We have adapted these results for the MSSM Higgs bosons to the NMSSM case. Furthermore, the resummed corrections have been included in the decays into a $\tau$ pair and into a strange quark pair. Details on the determination of these corrections are given in section 5.3 .

For the decays of the heavier neutral Higgs bosons into a top quark pair below the threshold off-shell decays can be sizable and have been included in the program. For the charged Higgs boson, off-shell decays below the top-bottom, the top-strange and the top-down quark threshold, respectively, have been taken into account. The decay widths have been obtained from [38] by making the necessary changes for the NMSSM case. In the charged Higgs boson decays into quarks we have taken into account generation mixing through the Cabibbo-Kobayashi-Maskawa (CKM) mixing matrix elements.

Decays into gluons: The decay width of a neutral Higgs boson into gluons, a loop-induced process already at tree-level, is mediated by quark loops and, in case of CP-even Higgs bosons, in addition by squark loops. The QCD corrections, which can be taken over from the SM, respectively, MSSM case, have been included up to $\mathrm{N}^{3} \mathrm{LO}$ in the limit of heavy quark [39 46] and squark [47] loop particle masses. The electroweak corrections are unknown for supersymmetric Higgs boson decays.

Decays into a pair of photons: Also the decay into a photon pair is loop-mediated already at lowest order, including $W$ boson, heavy fermion, charged Higgs boson, sfermion and chargino loops for the scalar Higgs boson decays and heavy fermion and chargino loops for the pseudoscalar ones [45]. The QCD corrections to quark and squark loops have been calculated including the full mass dependence both for the quarks [45, 55] and squarks [50]. These corrections can be taken over to the NMSSM and have been included in the program. The genuine SUSY-elw corrections for photonic SUSY Higgs decays are unknown.

Decays into $Z \gamma$ : The loop induced decays of scalar Higgs bosons into $Z \gamma$ are mediated by $W$, heavy fermion, charged Higgs, sfermion and chargino loops, while the pseudoscalar decays proceed only through charged fermion and chargino loops. While the electroweak corrections are unknown, the QCD corrections to quark loops are numerically small [57] and have not been taken into account.

Decays into massive gauge bosons: The decay width of the scalar NMSSM Higgs bosons into massive gauge bosons can be obtained from the SM decay width by replacing the SM Higgs coupling to gauge bosons by the corresponding NMSSM Higgs coupling. We have included the option of double off-shell decays [58] in the program. Electroweak corrections to the decay have not been calculated for the NMSSM case and are therefore not included. The pseudoscalar Higgs bosons do not decay into massive gauge bosons at tree-level.

\footnotetext{
${ }^{7}$ For the NLO QCD corrections including the full mass dependence, see [4, 45, and for the (SUSY-)QCD corrections including the full squark mass dependence, see [48 54 .

${ }^{8}$ Threshold effects have been discussed in [56].
} 
Decays into Higgs bosons: The heavier Higgs particles can decay into a pair of lighter Higgs bosons. Due to the enlarged Higgs sector various Higgs-to-Higgs decays are possible depending on the mass hierarchies [59]. The following decays have been included in the program $(j, k=1,2, l=1,2,3)$,

$$
\begin{aligned}
& H_{1} \rightarrow A_{1} A_{1}, \quad H_{1} \rightarrow A_{1} A_{2} \\
& H_{2} \rightarrow H_{1} H_{1}, \quad H_{2} \rightarrow A_{j} A_{k} \\
& H_{3} \rightarrow H_{j} H_{k}, \quad H_{3} \rightarrow A_{j} A_{k} \\
& A_{2} \rightarrow A_{1} H_{l} \text {. }
\end{aligned}
$$

The contributions from off-shell final states may be significant. We have therefore included double off-shell decays into two Higgs bosons with the Higgs bosons subsequently decaying into fermions. For $M_{H_{i}}>M_{H_{j}}$ and $M_{H_{i}}>M_{H_{k}}$ where $H_{i, j, k}$ denotes generically scalar and pseudoscalar Higgs bosons, the decay width is given by

$$
\begin{aligned}
\Gamma\left(H_{i} \rightarrow H_{j}^{*} H_{k}^{*}\right) & =\frac{1}{\pi^{2}} \int_{0}^{M_{H_{i}}^{2}} \frac{d Q_{1}^{2} M_{H_{j}} \Gamma_{H_{j}}}{\left(Q_{1}^{2}-M_{H_{j}}^{2}\right)^{2}+M_{H_{j}}^{2} \Gamma_{H_{j}}^{2}} \int_{0}^{\left(M_{H_{i}}-Q_{1}\right)^{2}} \frac{d Q_{2}^{2} M_{H_{k}} \Gamma_{H_{k}}}{\left(Q_{2}^{2}-M_{H_{k}}^{2}\right)^{2}+M_{H_{k}}^{2} \Gamma_{H_{k}}^{2}} \Gamma_{0} \\
\text { with } & \\
\Gamma_{0} & =\lambda_{H_{i} H_{j} H_{k}}^{2} \delta_{H} \frac{G_{F} M_{Z}^{4}}{16 \sqrt{2} \pi M_{H}} \lambda\left(Q_{1}^{2}, Q_{2}^{2} ; M_{H_{i}}^{2}\right) \frac{Q_{1}^{2} Q_{2}^{2}}{M_{H_{j}}^{2} M_{H_{k}}^{2}},
\end{aligned}
$$

where $\lambda_{H_{i} H_{j} H_{k}}$ denotes the trilinear Higgs coupling normalised to $\left(\sqrt{2} G_{F}\right)^{1 / 2} M_{Z}^{2}, \delta_{H}=2$ for $H_{j} \neq H_{k}$ and with the two-body phase space function

$$
\lambda(x, y, ; z)=\sqrt{(1-x / z-y / z)^{2}-4 x y / z^{2}} .
$$

Decays into a gauge and a Higgs boson: The Higgs bosons can also decay into a gauge and a Higgs boson, where in the NMSSM there is a plethora of possible decays, given by $(j=1,2, l=1,2,3)$

$$
\begin{array}{lllll}
H_{1} & \rightarrow A_{1} Z, & A_{1} & \rightarrow H_{j} Z \\
H_{2,3} & \rightarrow A_{j} Z, & H_{2,3} & \rightarrow H^{ \pm} W^{\mp} \\
A_{2} & \rightarrow H_{l} Z, & A_{2} & \rightarrow H^{ \pm} W^{\mp} \\
H^{ \pm} & \rightarrow H_{l} W^{ \pm}, & H^{ \pm} & \rightarrow A_{j} W^{ \pm} .
\end{array}
$$

The formulae for the decay widths can be easily obtained from the MSSM results (see e.g. 60] with the corresponding replacements of the involved couplings. These decays have been implemented in the program as well as the decays into a Higgs boson and an off-shell gauge boson which can be important. They have been obtained by adapting the MSSM formulae [38] accordingly.

Decays into SUSY particles: The decays into chargino or neutralino pairs [61,62] can reach branching ratios of up to $100 \%$ if they are kinematically allowed. They have been included in the program by adapting the MSSM Higgs couplings to neutralinos/charginos appropriately. Also the decays into sfermions of the third generation can become important if kinematically allowed [61]. We have included them for all generations. The SUSY-QCD corrections to the stop and sbottom decays of the MSSM Higgs bosons have been provided in [63, 64] and reanalysed in [65]. We have adapted them to the NMSSM decays and included them in the program.

\subsection{Decay Widths in the CP-violating NMSSM}

In this section we list the changes in the Higgs boson decays for the CP-violating NMSSM. We start with the neutral Higgs bosons and give explicit formulae for the loop-induced decays into gluons and into photons. 
Decays into gluons: We introduce the Feynman rules for the neutral CP-violating Higgs bosons $H_{i}$ $(i=1, \ldots, 5)$ to fermions as

$$
-\frac{i g m_{f}}{2 M_{W}}\left[g_{H_{i} f f}^{S}-i \gamma_{5} g_{H_{i} f f}^{P}\right],
$$

with the real coupling factors for up- and down-type fermions, respectively,

$$
g_{H_{i} f f}^{S}= \begin{cases}\frac{\mathcal{R}_{i 2}}{\sin \beta} & \text { for } f=\text { up-type } \\ \frac{\mathcal{R}_{i 1}}{\cos \beta} & \text { for } f=\text { down-type }\end{cases}
$$

and

$$
g_{H_{i} f f}^{P}=\left\{\begin{array}{ll}
\frac{\mathcal{R}_{i 4}}{\tan \beta} & \text { for } f=\text { up-type } \\
\mathcal{R}_{i 4} \tan \beta & \text { for } f=\text { down-type }
\end{array} .\right.
$$

Here $\mathcal{R}_{i j}(i, j=1, \ldots, 5)$ denote the elements of the matrix, which rotates the interaction states to the Higgs mass eigenstates as defined in Eqs. (23) and (24). In the gluonic decay width only the diagonal Higgs coupling to sfermions $\tilde{f}_{j}(j=1,2)$ appears, which is defined as

$$
-i g \frac{M_{Z}^{2}}{M_{W}} g_{H_{i} \tilde{f}_{j} \tilde{f}_{j}} .
$$

Note that the coupling factor $g_{H_{i} \tilde{f}_{j} \tilde{f}_{k}}$ for the coupling to two different sfermions $(j \neq k)$ can be complex in the CP-violating NMSSM. As the expression for $g_{H_{i} \tilde{f}_{j} \tilde{f}_{j}}$ is quite lengthy we refer the reader to the program code, where it can be found explicitly. With these coupling definitions the tree-level decay width into gluons can be cast into the form

$$
\begin{aligned}
\Gamma\left(H_{i} \rightarrow g g\right)=\frac{G_{F} \alpha_{s}^{2} M_{H_{i}}^{3}}{64 \sqrt{2} \pi^{3}} & \left(\left|\sum_{q} g_{H_{i} q q}^{S} A_{1 / 2}^{S}\left(\tau_{q}\right)+\sum_{\tilde{q}} \frac{M_{Z}^{2}}{m_{\tilde{q}}^{2}} g_{H_{i} \tilde{q} \tilde{q}} A_{0}\left(\tau_{\tilde{q}}\right)\right|^{2}\right. \\
& \left.+4\left|\sum_{q} g_{H_{i} q q}^{P} A_{1 / 2}^{P}\left(\tau_{q}\right)\right|^{2}\right)
\end{aligned}
$$

with $\tau_{x}=4 m_{x}^{2} / M_{H_{i}}^{2}(x=q, \tilde{q})$ and the loop functions [66]

$$
\begin{aligned}
A_{1 / 2}^{S}(\tau) & =2 \tau[1+(1-\tau) f(\tau)] \\
A_{1 / 2}^{P}(\tau) & =\tau f(\tau) \\
A_{0}(\tau) & =-\tau[1-\tau f(\tau)],
\end{aligned}
$$

where

$$
f(\tau)=\left\{\begin{array}{cc}
\arcsin ^{2}\left(\frac{1}{\sqrt{\tau}}\right) & \tau \geq 1 \\
-\frac{1}{4}\left[\ln \left(\frac{1+\sqrt{1-\tau}}{1-\sqrt{1-\tau}}\right)-i \pi\right]^{2} & \tau<1
\end{array} .\right.
$$

The coupling $\alpha_{s}$ is evaluated at the scale $M_{H_{i}}$. The sums are taken over the top, bottom and charm quarks and over all squark mass eigenstates. 
Decays into photons and into $Z \gamma$ : The decays into photons are mediated by charged particle loops. In addition to the Feynman rules introduced for the gluonic decay modes we have the Feynman rules for the Higgs couplings to the charged gauge bosons $W^{ \pm}$,

$$
i g M_{W} g_{H_{i} W W}, \quad \text { with } \quad g_{H_{i} W W}=\mathcal{R}_{i 1} \cos \beta+\mathcal{R}_{i 2} \sin \beta,
$$

and for the neutral Higgs couplings to a charged Higgs bosons pair,

$$
-\frac{i g M_{Z}^{2}}{2 M_{W}} g_{H_{i} H^{+} H^{-}} \text {. }
$$

The coupling factor, which is rather lengthy, can easily be read off from the program code. Furthermore, we define the Higgs couplings to a chargino pair as $(j=1,2) 9$

$$
-i \frac{g}{2}\left[g_{H_{i} \tilde{\chi}_{j}^{+} \tilde{\chi}_{j}^{-}}^{S}-i \gamma_{5} g_{H_{i} \tilde{\chi}_{j}^{+} \tilde{\chi}_{j}^{-}}^{P}\right]
$$

Only the diagonal couplings appear in the partial width. Their explicit form can be inferred from the program code. The leading order decay width into photons is then given by

$$
\begin{gathered}
\Gamma\left(H_{i} \rightarrow \gamma \gamma\right)=\frac{G_{F} \alpha^{2} M_{H_{i}}^{3}}{128 \pi^{3} \sqrt{2}}\left(\mid \sum_{f} N_{c f} e_{f}^{2} g_{H_{i} f f}^{S} A_{1 / 2}^{S}\left(\tau_{f}\right)+\sum_{\tilde{\chi}_{j}^{ \pm}} \frac{M_{W}}{M_{\tilde{\chi}_{j}^{ \pm}}} g_{H_{i} \tilde{\chi}_{j}^{+} \tilde{\chi}_{j}^{-}} A_{1 / 2}^{S}\left(\tau_{\tilde{\chi}_{j}^{ \pm}}\right)\right. \\
+g_{H_{i} W W} A_{1}\left(\tau_{W}\right)+\frac{M_{Z}^{2}}{2 M_{H^{ \pm}}^{2}} g_{H_{i} H^{+} H^{-}} A_{0}\left(\tau_{H^{ \pm}}\right)+\left.\sum_{\tilde{f}} N_{c f} e_{f}^{2} \frac{M_{Z}^{2}}{m_{\tilde{f}}^{2}} g_{H_{i} \tilde{f} \tilde{f}} A_{0}\left(\tau_{\tilde{f}}\right)\right|^{2} \\
\left.+4\left|\sum_{f} N_{c f} e_{f}^{2} g_{H_{i} f f}^{P} A_{1 / 2}^{P}\left(\tau_{f}\right)+\sum_{\tilde{\chi}_{j}^{ \pm}} \frac{M_{W}}{M_{\tilde{\chi}_{j}^{ \pm}}} g_{H_{i} \tilde{\chi}_{j}^{+} \tilde{\chi}_{j}^{-}}^{P} A_{1 / 2}^{P}\left(\tau_{\tilde{\chi}_{j}^{ \pm}}\right)\right|^{2}\right)
\end{gathered}
$$

The fermion sum proceeds over the top, charm and bottom quarks as well as the $\tau$ lepton. The sum over $\tilde{f}$ includes all squark and the stau mass eigenstates. With $N_{c f}=3,1$ we denote the colour factor of the (s)quarks and the (s)tau, respectively, and with $e_{f}$ their corresponding electric charge. The loop function $A_{1}$ is given by [67]

$$
A_{1}(\tau)=-(2+3 \tau+3 \tau(2-\tau) f(\tau)) .
$$

Analogously, the decay width into $Z \gamma$ can be derived from the decay widths of the scalar and pseudoscalar Higgs bosons in the real NMSSM with the appropriate replacements by the couplings as given in this subsection.

Other decay modes: In the decay widths into bosonic final states (gauge bosons, Higgs pairs, Higgs plus gauge boson pairs, sfermions) the respective coupling factors simply have to be replaced with the corresponding couplings of the CP-violating Higgs bosons. In case of complex valued couplings the absolute value squared has to be taken in the decay width. The decay widths into the fermionic final states (quark and charged fermion pairs as well as chargino and neutralino final states) are given by the incoherent sum of the scalar and pseudoscalar decay widths of the real NMSSM. In the decays into bottom and strange quarks as well as into tau leptons higher order SUSY corrections have been included through effective Yukawa couplings. The formulae are given in section 5.3 .

\footnotetext{
${ }^{9}$ Note that $g_{H_{i} \tilde{\chi}_{j}^{+} \tilde{\chi}_{j}^{-}}^{P}$ in the code is actually introduced as $i g_{H_{i} \tilde{\chi}_{j}^{+} \tilde{\chi}_{j}^{-}}^{P}$.
} 
Charged Higgs boson decays: The decays into quark and lepton pairs do not change with respect to the real NMSSM. The decay widths into a charged $W$ and neutral Higgs $H_{i}$ final state are derived from the CP-conserving case by adding up incoherently the decay widths into $W$ plus CP-even and $W$ plus CP-odd Higgs bosons, respectively. The decay widths into sfermions are the same as in the real NMSSM but with the respective coupling squared replaced by the absolute value squared of the now complex valued coupling. Also the charged Higgs couplings to a neutralino-chargino pair are in general complex. Defining the Feynman rule as

$$
-\frac{i g}{2}\left[a_{L} P_{L}+a_{R} P_{R}\right]
$$

with the projectors $P_{L, R}=\left(1 \mp \gamma_{5}\right) / 2$ and complex $a_{L}, a_{R}$ (cf. the program code for the explicit expressions), the decay width is given by

$$
\begin{aligned}
\Gamma\left(H^{ \pm} \rightarrow \tilde{\chi}_{j}^{ \pm} \tilde{\chi}_{k}^{0}\right)=\frac{G_{F} M_{W}^{2} \lambda\left(M_{\tilde{\chi}_{j}^{ \pm}}^{2}, M_{\tilde{\chi}_{k}^{0}}^{2} ; M_{H^{ \pm}}^{2}\right)}{2 \sqrt{2} \pi M_{H^{ \pm}}}\left[\left(\left|a_{L}\right|^{2}+\left|a_{R}\right|^{2}\right)\left(M_{H^{ \pm}}^{2}-M_{\tilde{\chi}_{j}^{ \pm}}^{2}-M_{\tilde{\chi}_{k}^{0}}^{2}\right)\right. & \left.-4 \operatorname{Re}\left(a_{L}^{*} a_{R}\right) M_{\tilde{\chi}_{j}^{ \pm}} M_{\tilde{\chi}_{k}^{0}}\right],
\end{aligned}
$$

with $j=1,2$ and $k=1, \ldots, 5$ and the two-body phase space function $\lambda(x, y ; z)$ given in Eq. (33).

\subsection{SUSY corrections to decays into fermions in the real and in the complex NMSSM}

The leading parts of the SUSY-QCD and SUSY-elw corrections to the decays into a bottom quark pair can be taken into account by absorbing them into effective bottom Yukawa couplings. The leading corrections can be obtained from an effective Lagrangian [24, 25] and can be taken over from the MSSM case by deriving the effective Lagrangian for the NMSSM Higgs sector. In the real NMSSM it is given by

$$
\mathcal{L}_{e f f}=-y_{b} \bar{b}_{R}\left[\left(1+\Delta_{1}\right) H_{d}^{0}+\frac{\lambda\left(1+\Delta_{1}\right) \Delta_{b}}{\mu_{\mathrm{eff}} \tan \beta} S^{*} H_{u}^{0 *}\right] b_{L}+\text { h.c. }
$$

where $H_{d(u)}^{0}$ are the neutral components of the doublet fields coupling to down-type (up-type) quarks. The corrections $\Delta_{b}$ and $\Delta_{1}$ include the SUSY-QCD and SUSY-elw corrections and induce a modification of the relation between the bottom quark mass $m_{b}$ and the Yukawa coupling $y_{b}$. After expanding the Lagrangian to include the higher order corrections we have

$$
\begin{aligned}
\mathcal{L}_{\text {eff }}=-m_{b} \bar{b}[1- & \left.i \gamma_{5} \frac{G}{v}\right] b \\
-\frac{m_{b} / v}{1+\Delta_{b}} \bar{b} & \left\{\left[\frac{\mathcal{R}_{11}^{S}}{\cos \beta}\left(1+\Delta_{b} \frac{\mathcal{R}_{12}^{S}}{\mathcal{R}_{11}^{S}} \frac{1}{\tan \beta}\right)+\left(\frac{\mathcal{R}_{13}^{S} v}{v_{s}}\right) \Delta_{b}\right] H_{1}\right. \\
& +\left[\frac{\mathcal{R}_{21}^{S}}{\cos \beta}\left(1+\Delta_{b} \frac{\mathcal{R}_{22}^{S}}{\mathcal{R}_{21}^{S}} \frac{1}{\tan \beta}\right)+\left(\frac{\mathcal{R}_{23}^{S} v}{v_{s}}\right) \Delta_{b}\right] H_{2} \\
+ & {\left[\frac{\mathcal{R}_{31}^{S}}{\cos \beta}\left(1+\Delta_{b} \frac{\mathcal{R}_{32}^{S}}{\mathcal{R}_{31}^{S}} \frac{1}{\tan \beta}\right)+\left(\frac{\mathcal{R}_{33}^{S} v}{v_{s}}\right) \Delta_{b}\right] H_{3} } \\
& -\left[\mathcal{R}_{11}^{P} \tan \beta\left(1-\Delta_{b} \frac{1}{\tan ^{2} \beta}\right)-\left(\frac{\mathcal{R}_{12}^{P} v}{v_{s}}\right) \Delta_{b}\right] i \gamma_{5} A_{1} \\
& \left.-\left[\mathcal{R}_{21}^{P} \tan \beta\left(1-\Delta_{b} \frac{1}{\tan ^{2} \beta}\right)-\left(\frac{\mathcal{R}_{22}^{P} v}{v_{s}}\right) \Delta_{b}\right] i \gamma_{5} A_{2}\right\} b,
\end{aligned}
$$


where we have used

$$
\begin{aligned}
m_{b} & =\frac{y_{b} v_{d}}{\sqrt{2}}\left(1+\Delta_{b}\right)\left(1+\Delta_{1}\right) \\
H_{d}^{0} & =\frac{1}{\sqrt{2}}\left[v_{d}+\mathcal{R}_{11}^{S} H_{1}+\mathcal{R}_{21}^{S} H_{2}+\mathcal{R}_{31}^{S} H_{3}+i \mathcal{R}_{11}^{P} \sin \beta A_{1}+i \mathcal{R}_{21}^{P} \sin \beta A_{2}+i G \cos \beta\right] \\
H_{u}^{0} & =\frac{1}{\sqrt{2}}\left[v_{u}+\mathcal{R}_{12}^{S} H_{1}+\mathcal{R}_{22}^{S} H_{2}+\mathcal{R}_{32}^{S} H_{3}+i \mathcal{R}_{11}^{P} \cos \beta A_{1}+i \mathcal{R}_{21}^{P} \cos \beta A_{2}-i G \sin \beta\right] \\
S & =\frac{1}{\sqrt{2}}\left[v_{s}+\mathcal{R}_{13}^{S} H_{1}+\mathcal{R}_{23}^{S} H_{2}+\mathcal{R}_{33}^{S} H_{3}+i \mathcal{R}_{12}^{P} A_{1}+i \mathcal{R}_{22}^{P} A_{2}\right]
\end{aligned}
$$

with the mixing matrices $\mathcal{R}^{S}$ and $\mathcal{R}^{P}$ defined in Eq. (6) and (7), respectively. The correction $\Delta_{b}$ contains the one-loop SUSY-QCD and SUSY-elw corrections,

$$
\begin{aligned}
\Delta_{b} & =\frac{\Delta_{b}^{Q C D}+\Delta_{b}^{e l w}}{1+\Delta_{1}} \\
\Delta_{b}^{Q C D} & =\Delta_{b}^{Q C D(1)} \\
\Delta_{b}^{e l w} & =\Delta_{b}^{e l w(1)}
\end{aligned}
$$

with the one-loop corrections given by

$$
\begin{aligned}
\Delta_{b}^{Q C D(1)} & =\frac{C_{F}}{2} \frac{\alpha_{s}\left(\mu_{R}\right)}{\pi} m_{\tilde{g}} \mu_{\mathrm{eff}} \tan \beta I\left(m_{\tilde{b}_{1}}^{2}, m_{\tilde{b}_{2}}^{2}, m_{\tilde{g}}^{2}\right) \\
\Delta_{b}^{e l w(1)} & =\frac{y_{t}^{2}\left(\mu_{R}\right)}{(4 \pi)^{2}} A_{t} \mu_{\mathrm{eff}} \tan \beta I\left(m_{\tilde{t}_{1}}^{2}, m_{\tilde{t}_{2}}^{2}, \mu_{\mathrm{eff}}^{2}\right)
\end{aligned}
$$

where $y_{t}=\sqrt{2} m_{t} /(v \sin \beta)$ denotes the top-Yukawa coupling, $C_{F}=4 / 3$, and

$$
\Delta_{1}=-\frac{C_{F}}{2} \frac{\alpha_{s}\left(\mu_{R}\right)}{\pi} m_{\tilde{g}} A_{b} I\left(m_{\tilde{b}_{1}}^{2}, m_{\tilde{b}_{2}}^{2}, m_{\tilde{g}}^{2}\right) .
$$

The generic function $I$ is defined as

$$
I(a, b, c)=\frac{a b \log \frac{a}{b}+b c \log \frac{b}{c}+c a \log \frac{c}{a}}{(a-b)(b-c)(a-c)} .
$$

Note that the scale of $\alpha_{s}$ in the SUSY-QCD corrections has been set equal to $\mu_{R}=\left(m_{\tilde{b}_{1}}+m_{\tilde{b}_{2}}+\right.$ $\left.\left|M_{\tilde{g}}\right|\right) / 3$, while in the SUSY-elw corrections it is $\mu_{R}=\left(m_{\tilde{t}_{1}}+m_{\tilde{t}_{2}}+|\mu|\right) / 3$, each $\alpha_{s}$ calculated with five active flavours. The higher order corrected decay width of the NMSSM Higgs bosons $\Phi=H_{1}, H_{2}, H_{3}, A_{1}, A_{2}$ into $b \bar{b}$, including QCD and SUSY-QCD corrections, can be cast into the form [25]

$$
\Gamma(\Phi \rightarrow b \bar{b})=\frac{3 G_{F} M_{\Phi}}{4 \sqrt{2} \pi} \bar{m}_{b}^{2}\left(M_{\Phi}\right)\left[\Delta_{\mathrm{QCD}}+\Delta_{t}^{\Phi}\right] \tilde{g}_{b}^{\Phi}\left[\tilde{g}_{b}^{\Phi}+\Delta_{S Q C D}^{r e m}\right]
$$

The logarithmically enhanced part of the QCD corrections has been absorbed in the running $\overline{\mathrm{MS}}$ bottom quark mass $\bar{m}_{b}\left(M_{\Phi}\right)$ at the corresponding Higgs mass scale $M_{\Phi}$. The QCD corrections $\Delta_{Q C D}$ and the top quark induced corrections $\Delta_{t}^{\Phi}$ can be taken over from the MSSM case [33 35$]$ 
by adapting the Higgs couplings and read10

$$
\begin{aligned}
\Delta_{\mathrm{QCD}}= & 1+5.67 \frac{\alpha_{s}\left(M_{\Phi}\right)}{\pi}+\left(35.94-1.36 N_{F}\right)\left(\frac{\alpha_{s}\left(M_{\Phi}\right)}{\pi}\right)^{2} \\
& +\left(164.14-25.77 N_{F}+0.259 N_{F}^{2}\right)\left(\frac{\alpha_{s}\left(M_{\Phi}\right)}{\pi}\right)^{3} \\
\Delta_{t}^{\phi_{S}}= & \frac{g_{t}^{\phi_{S}}}{g_{b}^{\phi_{S}}}\left(\frac{\alpha_{s}\left(M_{\phi_{S}}\right)}{\pi}\right)^{2}\left[1.57-\frac{2}{3} \log \frac{M_{\phi_{S}}^{2}}{M_{t}^{2}}+\frac{1}{9} \log ^{2} \frac{\bar{m}_{b}^{2}\left(M_{\phi_{S}}\right)}{M_{\phi_{S}}^{2}}\right] \\
\Delta_{t}^{\phi_{P}}= & \frac{g_{t}^{\phi_{P}}}{g_{b}^{\phi_{P}}}\left(\frac{\alpha_{s}\left(M_{\phi_{P}}\right)}{\pi}\right)^{2}\left[3.83-\log \frac{M_{\phi_{P}}^{2}}{M_{t}^{2}}+\frac{1}{6} \log ^{2} \frac{\bar{m}_{b}^{2}\left(M_{\phi_{P}}\right)}{M_{\phi_{P}}^{2}}\right]
\end{aligned}
$$

with $\phi_{S} \equiv H_{1}, H_{2}, H_{3}, \phi_{P} \equiv A_{1}, A_{2}$ and where $N_{F}=5$ active flavors are taken into account. The coupling factors $g_{b}^{\Phi}\left(g_{t}^{\Phi}\right)$ with respect to the SM Higgs-bottom (top) Yukawa coupling are given by

$$
\begin{aligned}
& g_{t}^{H_{i}}=\mathcal{R}_{i 2}^{S} / \sin \beta, \quad g_{b}^{H_{i}}=\mathcal{R}_{i 1}^{S} / \cos \beta, \quad i=1,2,3, \\
& g_{t}^{A_{j}}=\mathcal{R}_{j 1}^{P} / \tan \beta, \quad g_{b}^{A_{j}}=\mathcal{R}_{j 1}^{P} \tan \beta, \quad j=1,2 .
\end{aligned}
$$

The dominant part of the SUSY-QCD [25, 64, 68] corrections has been absorbed in the effective Yukawa couplings $\tilde{g}_{b}^{\Phi}$. Adapting the results from the MSSM to the NMSSM they read

$$
\begin{aligned}
& \tilde{g}_{b}^{H_{1}}=\frac{g_{b}^{H_{1}}}{1+\Delta_{b}}\left[1+\Delta_{b}\left(\frac{\mathcal{R}_{12}^{S}}{\mathcal{R}_{11}^{S} \tan \beta}+\frac{\mathcal{R}_{13}^{S} v \cos \beta}{\mathcal{R}_{11}^{S} v_{s}}\right)\right] \\
& \tilde{g}_{b}^{H_{2}}=\frac{g_{b}^{H_{2}}}{1+\Delta_{b}}\left[1+\Delta_{b}\left(\frac{\mathcal{R}_{22}^{S}}{\mathcal{R}_{21}^{S} \tan \beta}+\frac{\mathcal{R}_{23}^{S} v \cos \beta}{\mathcal{R}_{21}^{S} v_{s}}\right)\right] \\
& \tilde{g}_{b}^{H_{3}}=\frac{g_{b}^{H_{3}}}{1+\Delta_{b}}\left[1+\Delta_{b}\left(\frac{\mathcal{R}_{32}^{S}}{\mathcal{R}_{31}^{S} \tan \beta}+\frac{\mathcal{R}_{33}^{S} v \cos \beta}{\mathcal{R}_{31}^{S} v_{s}}\right)\right]
\end{aligned}
$$

and

$$
\begin{aligned}
& \tilde{g}_{b}^{A_{1}}=\frac{g_{b}^{A_{1}}}{1+\Delta_{b}}\left[1+\Delta_{b}\left(-\frac{1}{\tan ^{2} \beta}-\frac{\mathcal{R}_{12}^{P} v}{\mathcal{R}_{11}^{P} v_{S} \tan \beta}\right)\right] \\
& \tilde{g}_{b}^{A_{2}}=\frac{g_{b}^{A_{2}}}{1+\Delta_{b}}\left[1+\Delta_{b}\left(-\frac{1}{\tan ^{2} \beta}-\frac{\mathcal{R}_{22}^{P} v}{\mathcal{R}_{21}^{P} v_{S} \tan \beta}\right)\right] .
\end{aligned}
$$

The remaining part of the SUSY-QCD corrections, after the main corrections have been absorbed in the effective bottom Yukawa couplings $\tilde{g}_{b}^{\Phi}$, is given by the remainder $\triangle_{S Q C D}^{r e m}$. The decay width of Eq. (62) has been implemented in the decay program.

The SUSY-QCD corrections at one-loop order have also been included in the decays into strange quarks, i.e. $\Delta_{b}$ of Eq. (55) for these decays is replaced by

$$
\Delta_{s}=\left.\frac{\Delta_{b}^{Q C D(1)}}{1+\Delta_{1}}\right|_{b \rightarrow s}
$$

\footnotetext{
${ }^{10}$ Note that actually in the code we have programmed the QCD corrections for the completely massive case at next-to-leading order, translated to the $\overline{\mathrm{MS}}$ scheme, and interpolated with the massless expression for large Higgs masses, according to the implementation in HDECAY [22].
} 
with $\Delta_{b}^{Q C D(1)}$ and $\Delta_{1}$ obtained from Eqs. (58) and (60) after substituting $A_{b}, m_{\tilde{b}_{1,2}}$ with $A_{s}, m_{\tilde{s}_{1,2}}$. For the decays into lepton finals states $l=e, \mu, \tau$ the SUSY corrections have been included in the decay widths by absorbing them into the effective couplings $\tilde{g}_{l}^{\Phi}$,

$$
\Gamma(\Phi \rightarrow l l)=\frac{G_{F}}{4 \pi \sqrt{2}} M_{\Phi} m_{l}^{2}\left(\tilde{g}_{l}^{\Phi}\right)^{2}
$$

The effective couplings $\tilde{g}_{l}^{\Phi}$ are defined as in Eqs. (66)-(70) by replacing $b$ with $l$ and with $\Delta_{l}$ given by 23.

$$
\Delta_{l}=\frac{\alpha_{1}}{4 \pi} M_{1} \mu_{\mathrm{eff}} \tan \beta I\left(m_{\tilde{l}_{1}}^{2}, m_{\tilde{l}_{2}}^{2}, M_{1}^{2}\right)+\frac{\alpha_{2}}{4 \pi} M_{2} \mu_{\mathrm{eff}} \tan \beta I\left(m_{\tilde{\nu}_{l}}^{2}, M_{2}^{2}, \mu_{\mathrm{eff}}^{2}\right)
$$

with $\alpha_{1,2}=g_{1,2}^{2} / 4 \pi$.

The SUSY-QCD and SUSY-elw corrections to the charged Higgs boson decays $H^{ \pm}$into up bottom, charm bottom and top bottom final states, respectively, have been included analogously to the decays of the neutral Higgs bosons $\Phi$ into $b \bar{b}$, Eq. (62), with $\Delta_{\mathrm{QCD}}$ given in [37] and $\Delta_{t}^{\Phi}=0$. The effective coupling $\tilde{g}_{b}^{H^{ \pm}}$reads

$$
\tilde{g}_{b}^{H^{ \pm}}=\frac{g_{b}^{H^{ \pm}}}{1+\Delta_{b}}\left[1-\frac{\Delta_{b}}{\tan ^{2} \beta}\right]
$$

with

$$
g_{b}^{H^{ \pm}}=\tan \beta
$$

and $\Delta_{b}$ given in Eq. (55). The SUSY-QCD corrections have been taken into account in the decays of $H^{ \pm}$into a strange quark with an up, charm and top quark, respectively, by replacing $\Delta_{b}$ of Eq. (55) with $\Delta_{s}$ of Eq. (71). Finally, the SUSY corrections are implemented in the $H^{ \pm}$decays into $l \nu_{l}(l=e, \mu, \tau)$ via the effective couplings given in terms of $\Delta_{l}$.

In the complex NMSSM the leading parts of the SUSY-QCD and SUSY-elw corrections in the decays into bottom quarks are derived from the effective Lagrangian

$$
\mathcal{L}_{e f f}=-y_{b} \bar{b}_{R}\left[\left(1+\Delta_{1}\right) H_{d}^{0}+\frac{\lambda^{*} e^{i \varphi_{u}}\left(1+\Delta_{1}\right) \Delta_{b}}{\mu_{\mathrm{eff}}^{*} \tan \beta} S^{*} H_{u}^{0 *}\right] b_{L}+\text { h.c. } .
$$

Introducing the neutral components of the two doublets and singlet fields as

$$
\begin{aligned}
H_{d}^{0} & =\frac{1}{\sqrt{2}}\left[v_{d}+\sum_{j=1}^{5}\left(\mathcal{R}_{j 1}+i \sin \beta \mathcal{R}_{j 4}\right) H_{j}+i \cos \beta G\right], \\
H_{u}^{0} & =\frac{e^{i \varphi_{u}}}{\sqrt{2}}\left[v_{u}+\sum_{j=1}^{5}\left(\mathcal{R}_{j 2}+i \cos \beta \mathcal{R}_{j 4}\right) H_{j}-i \sin \beta G\right], \\
S & =\frac{e^{i \varphi_{s}}}{\sqrt{2}}\left[v_{s}+\sum_{j=1}^{5}\left(\mathcal{R}_{j 3}+i \mathcal{R}_{j 5}\right) H_{j}\right]
\end{aligned}
$$

in Eq. (76), the Lagrangian reads

$$
\left.\mathcal{L}_{e f f}=-m_{b} \bar{b}\left(1-i \gamma_{5} \frac{G}{v}\right) b-\frac{m_{b}}{v} \sum_{j=1}^{5} \bar{b}\left[\tilde{g}_{b L}^{H_{j}} P_{L}+\left(\tilde{g}_{b L}^{H_{j}}\right)^{*} P_{R}\right)\right] H_{j} b
$$


with

$$
\tilde{g}_{b L}^{H_{j}}=\frac{1}{\left(1+\Delta_{b}\right)}\left[\frac{\mathcal{R}_{j 1}}{\cos \beta}+\frac{\mathcal{R}_{j 2}}{\sin \beta} \Delta_{b}+\frac{\mathcal{R}_{j 3} v}{v_{s}} \Delta_{b}+i \mathcal{R}_{j 4} \tan \beta\left(1-\frac{\Delta_{b}}{\tan ^{2} \beta}\right)-i \frac{\mathcal{R}_{j 5} v}{v_{s}} \Delta_{b}\right] .
$$

The rotation matrix $\mathcal{R}$ has been defined in Eq. (23). The correction $\Delta_{b}$ includes the one-loop SUSY-QCD and SUSY-elw corrections which in the complex NMSSM are given by

$$
\begin{aligned}
\Delta_{b} & =\frac{\Delta_{b}^{Q C D(1)}+\Delta_{b}^{e l w(1)}}{1+\Delta_{1}}, \\
\Delta_{b}^{Q C D(1)} & =\frac{C_{F}}{2} \frac{\alpha_{s}\left(\mu_{R}\right)}{\pi} M_{3}^{*} \mu_{\mathrm{eff}}^{*} \tan \beta I\left(m_{\tilde{b}_{1}}^{2}, m_{\tilde{b}_{2}}^{2}, m_{\tilde{g}}^{2}\right), \\
\Delta_{b}^{e l w(1)} & =\frac{y_{t}^{2}\left(\mu_{R}\right)}{(4 \pi)^{2}} A_{t}^{*} \mu_{\mathrm{eff}}^{*} \tan \beta I\left(m_{\tilde{t}_{1}}^{2}, m_{\tilde{t}_{2}}^{2},\left|\mu_{\mathrm{eff}}\right|^{2}\right), \\
\Delta_{1} & =-\frac{C_{F}}{2} \frac{\alpha_{s}}{\pi} M_{3}^{*} A_{b} I\left(m_{\tilde{b}_{1}}^{2}, m_{\tilde{b}_{2}}^{2}, m_{\tilde{g}}^{2}\right) .
\end{aligned}
$$

The corrections $\Delta_{b}$ and $\Delta_{1}$ are in general complex and depend on the phases of the gaugino mass $M_{3}$, of the trilinear couplings $A_{t}, A_{b}$ and on the phase of the effective Higgs mixing parameter, which in the CP-violating NMSSM is given by $\mu_{\mathrm{eff}}=\lambda v_{s} e^{i \varphi_{s}} / \sqrt{2}$.

The decay width of the Higgs boson $\Phi=H_{j}(j=1, \ldots, 5)$ into $b \bar{b}$, in the complex NMSSM can be written as

$$
\Gamma(\Phi \rightarrow b \bar{b})=\frac{3 G_{F} M_{\Phi}}{4 \sqrt{2} \pi} \bar{m}_{b}^{2}\left(M_{\Phi}\right)\left[\Delta_{\mathrm{QCD}}+\Delta_{t}^{\Phi}\right]\left|\tilde{g}_{b L}^{\Phi}\right|^{2}
$$

Note, in particular, that contrary to the real NMSSM we do not include a remainder $\Delta_{S Q C D}^{r e m}$ in the decay width, as it is not available at present. This leads, when using the program package for the complex NMSSM in the limit of the real NMSSM to differences in the decay widths into $b$-quark pairs with respect to the results obtained from the program package for the real NMSSM. The differences are below the percent level 11 The one-loop SUSY-QCD corrections to the decays into strange quarks are obtained after substituting $\Delta_{b}$ as given in Eq. (82) with $\Delta_{s}=\Delta_{b}^{Q C D(1)} /(1+$ $\left.\Delta_{1}\right)\left.\right|_{b \rightarrow s}$. For the decays into leptons $\Delta_{b}$ has to be replaced with $\Delta_{l}$, where $\Delta_{l}$ in the complex case is given by Eq. (73) with $M_{1}, M_{2}$ and $\mu_{\text {eff }}$ replaced by $M_{1}^{*}, M_{2}^{*}$ and $\mu_{\text {eff }}^{*}$ and $M_{1}^{2}, M_{2}^{2}, \mu_{\text {eff }}^{2}$ by $\left|M_{1}\right|^{2}$, $\left|M_{2}\right|^{2},\left|\mu_{\mathrm{eff}}\right|^{2}$.

The effective coupling (including the $\Delta_{b}$ effect) of the charged Higgs boson to a top-bottom quark pair can be read off from the Lagrangian

$$
\mathcal{L}_{\text {eff }}=\frac{\sqrt{2}}{v}\left(V_{t b}^{\mathrm{CKM}}\right)^{*} \bar{b}\left[m_{b} \tan \beta \frac{1-\Delta_{b} / \tan ^{2} \beta}{1+\Delta_{b}} P_{L}+\frac{m_{t}}{\tan \beta} P_{R}\right] H^{-} t+\text { h.c. },
$$

where $V^{\text {CKM }}$ denotes the CKM matrix and the phase $\varphi_{u}$ has been included in the CKM matrix element through a factor $e^{i \varphi_{u} / 2}$.

\section{Program Description}

The program package consists of a wrap file called nmssmcalc.f and three main files:

\footnotetext{
${ }^{11}$ Further differences appear in the decays into stop and sbottom pairs, respectively, as in the complex case we do not include SUSY-QCD corrections.
} 
1. CalcMasses.F for the calculation of the one-loop corrected NMSSM Higgs boson masses in the real and the complex NMSSM;

2. bhdecay. $f$ for the calculation of the NMSSM Higgs boson decay widths and branching ratios in the real NMSSM;

3. bhdecay_c.f for the calculation of the decay widths and branching ratios in the complex NMSSM.

There are additional files containing subroutines needed for the calculation of the decay widths. All files are written in Fortran. The package needs two input files:

1. an input file in the SLHA format with default name inp. dat, which is read in by nmssmcalc.f;

2. an input file bhdecay. in which is read in by bhdecay(_c).f and which contains the setting of the CKM parameters as well as of several flags for the decay calculation. They are specified in Appendix $\mathrm{A}$.

The mass routine CalcMasses.F provides an output file in the SLHA format, which is read in by the decay routine bhdecay(_c).f which in turn writes out the results in an SLHA file. The whole package is compiled with the help of a makefile. In the following the various files and their functions will be described as well as the compilation and the running of the program package.

nmssmcalc.f: This wrap file reads in the input parameters needed for the calculation of the oneloop corrected NMSSM Higgs boson masses. The input file which has the default name inp. dat, must be provided in the SLHA format and has to contain the blocks MODSEL, SMINPUTS, MINPAR and EXTPAR, as well as IMEXTPAR and CMPLX in the complex case, with the related input parameters. The required parameters are described in more detail in the following section 7 . The file then calls CalcMasses.F by transferring the parameters which have been read in, so that the routine can calculate the one-loop corrections of the NMSSM Higgs boson masses and writes them out in an SLHA output file with default name slha.in. Subsequently nmssmcalc.f calls bhdecay.f, in case the CP-conserving NMSSM has been chosen in the input file and bhdecay_c.f in case of a complex NMSSM choice, respectively. These routines read in the output file provided by CalcMasses.F and calculate the Higgs decay widths and branching ratios in the framework of the CP-conserving or CP-violating NMSSM with the results written out in an SLHA output file (default name slha_decay.out). The user can also specify the names of the input file and of the output files provided by the mass and decay routines in the command line when running the program.

CalcMasses.F: This Fortran code contains the subroutine CalcMasses for the calculation the one-loop corrections to the NMSSM Higgs boson masses in the CP-conserving or CP-violating NMSSM. The renormalisation schemes applied are a mixture of on-shell and $\overline{\mathrm{DR}}$ conditions, which are described in detail in Refs. [10,11] and have been briefly sketched in sections 3 and 4 . As described in sections 3 and 4 the schemes have been slightly modified in order to match the requirements of the SLHA (see also section 7). The subroutine calls further subroutines for the calculation of all NMSSM tree-level masses, of the one-loop Higgs masses, of the Higgs couplings, of the counterterms etc. They are listed together with their functions in the header of CalcMasses.F. At the end of the calculation it provides an SLHA output file slha.in. This file contains in particular the loop-corrected Higgs boson masses with the related Higgs mixing parameters, the NMSSM SUSY particle spectrum with corresponding mixing matrices as well as further blocks needed for the calculation of the decay widths.

bhdecay.f, bhdecay_c.f: The subroutines contained in these Fortran codes calculate the decay widths and branching ratios of the neutral and charged NMSSM Higgs bosons in the CP-conserving 
and the CP-violating case, respectively. They closely follow the structure and approximations of the Fortran code HDECAY version 6.00 [22]12, which calculates the Higgs decay widths and branching ratios in the SM and the MSSM including the most important higher order corrections. Thus the higher order corrections have been taken over wherever they can be adapted to the NMSSM case. This also means that a lot of common blocks and routines inherent in HDECAY have been retained. They are dubbed by the suffix _HDEC. Routines and common blocks that have been extended to the NMSSM case or are specific to the NMSSM case are denoted by the suffix _NMSSM, respectively _CNMSSM in the CP violating NMSSM. These Fortran routines are supplemented by various Fortran files taken over from HDECAY and adapted to the NMSSM where necessary: dmb.f (contains the 2loop SUSY-QCD and SUSY-elw corrections to the bottom Yukawa coupling in the real MSSM 13 ), hgaga.f (needed for the QCD corrections to the decays into photons), slha_nmssm.f (for reading in the SLHA input file) and in case of the real NMSSM also hsqsq_nmssm.f (called for the SUSYQCD corrections to decays into squark pairs). Finally Xvegas.f has been linked, which is used in the numerical integration of some off-shell decays.

The general structure of bhdecay (_c).f is the following: After calling the subroutine read_(c)nmssm.f to read in the input files bhdecay.in and slha.in it calls the core routine for the decays, hdec_(c)nmssm, in which the decay widths are calculated. This routine calls several other help routines. Afterwards write_(c)nmssm is called to write out the results in an SLHA output file.

How to compile and run the program package: The program package is compiled with the help of a makefile by typing make. This provides an executable file called run. The user has the choice to provide the names of the input and output files for CalcMasses.F (first and second argument) and the name of the output file provided by the decay routine (third argument) in the command line. Hence the command will be run name_file1 name_file2 name_file3 in this case.

Webpage of the program package: The program package can be downloaded from the url:

http://www.itp.kit.edu/ maggie/NMSSMCALC/

This webpage also contains a short description of the program. Furthermore, it informs about changes and updates of the program package.

\section{The SUSY Les Houches Accord}

The SUSY Les Houches Accord [29] has been extended in SLHA2 [30] to include the case of the NMSSM as well as possible CP-violation in the MSSM. In the following we list issues of our program package related to SLHA2 and how we treated them. Furthermore the parameters are specified, that have to be given by the user for the calculation of the loop-corrected mass values and the calculation of the branching ratios in the CP-conserving and CP-violating NMSSM.

The $2 \times 3$ mixing matrix $P$ for the rotation into the pseudoscalar mass eigenstates defined in the SLHA2 for the CP-conserving NMSSM differs from the matrix $\mathcal{R}^{P}$ rotating the imaginary parts of the interaction states to the CP-odd mass eigenstates, $c f$. Eq. (7). The tree-level matrix elements are related through

$$
\begin{array}{ll}
\mathcal{R}_{11}^{P}=P_{11} / \sin \beta_{n}=P_{12} / \cos \beta_{n} & \mathcal{R}_{12}^{P}=P_{13} \\
\mathcal{R}_{21}^{P}=P_{21} / \sin \beta_{n}=P_{22} / \cos \beta_{n} & \mathcal{R}_{22}^{P}=P_{23} .
\end{array}
$$

\footnotetext{
${ }^{12}$ See 69 for the extension of HDECAY to effective Lagrangians for a light Higgs-like scalar.

${ }^{13}$ These 2-loop corrections have not been adapted to the NMSSM yet, and therefore are not included in the NMSSM decays at present. This will be done in a future updated version of the program so that we include this routine already now in the program package.
} 
The angle $\beta_{n}$ is the rotation angle of the matrix $\mathcal{R}^{G}$ separating the massless Goldstone boson. It coincides at tree-level with the angle $\beta$ defined through the ratio of the VEVs $v_{u}$ and $v_{d}, \tan \beta=$ $v_{u} / v_{d}$.

In the block MODSEL the user has to specify the model to be used and if CP-violation is to be included or not,

BLOCK MODSEL

31 \# NMSSM

50 \# (0: CP conservation; 2: general CP violation)

Furthermore, the block SMINPUTS has to be provided as well as, in the block MINPAR, the value for $\tan \beta$. Note that in the input file inp. dat, which is read in by nmssmcalc.f, the block SMINPUTS has been extended by the $W$ boson pole mass as this mass value is used in the calculation of the loop-corrected Higgs boson masses and of the decay widths. It has to be given in the 9th entry of the block.

If working in the CP-conserving case it is sufficient to supply the block EXTPAR. The CP-violating case also requires the block IMEXTPAR which provides the imaginary parts to the corresponding real parts. In these two blocks the gaugino soft SUSY breaking mass parameters $M_{1}, M_{2}$ and $M_{3}$ have to be given and the soft SUSY breaking mass parameters for the 3rd generation, $m_{Q_{3}}, m_{t_{R}}, m_{b_{R}}, m_{L_{3}}$ and $m_{\tau_{R}} 14$. Also the soft SUSY breaking trilinear couplings $A_{t}, A_{b}, A_{\tau}, A_{\lambda}$ and $A_{\kappa}$, the NMSSM couplings $\lambda$ and $\kappa$ and the effective $\mu$ parameter, $\mu_{\text {eff }}$, have to be set. Together with $\lambda$ the latter value determines the value of $v_{s}$, and in the complex case also of $\varphi_{s}$, according to $\mu_{\text {eff }}=\lambda v_{s} e^{i \varphi_{s}} / \sqrt{2}$. The values of the soft SUSY breaking trilinear couplings of the second generation can be specified as well. If they are not given, they are set equal to the corresponding values of the third generation. The same holds for the first generation values: unless given they are set equal to the corresponding values of the second generation. The soft SUSY breaking masses of the first two generations are set equal to the corresponding values of the third generation, if not included in the SLHA input.

In our calculation of the loop-corrected masses we retain the option to either use $M_{H^{ \pm}}$or $\operatorname{Re} A_{\lambda}$. Depending on which input value is supplied by the user the according renormalisation scheme, as described in sections 3.2 and 4.2, is chosen for the calculation of the loop corrected masses. If the mass of the charged Higgs boson is chosen as an on-shell input the real part of the corresponding $A_{\lambda}$ value in the $\overline{\mathrm{DR}}$ scheme is calculated, which is needed in the SLHA output file in accordance with the SLHA conventions. Furthermore the $\overline{\mathrm{DR}}$ values of the imaginary parts of $A_{\lambda}$ and $A_{\kappa}$ are also supplied in the output file for the CP-violating case.

In the block EXTPAR the user has the option to give the renormalisation scale at which the parameters are evaluated. If this value is not given the renormalisation scale $\mu_{\text {ren }}$ is set equal to the geometric mean of the soft SUSY breaking masses in the stop sector,

$$
\mu_{\mathrm{ren}}=\sqrt{m_{Q_{3}} m_{t_{R}}} \text {. }
$$

At present this scale is only used to set the renormalisation scale in the mass calculation.

In SLHA2 there is no block foreseen for complex phases in the Higgs sector. We therefore added a new block called CMPLX where the value of the phase $\varphi_{u}$ can be specified according to

BLOCK CMPLX

3 \# phiu

We also had to add a new block containing the matrix elements of the $5 \times 5$ Higgs mixing matrix of the CP-violating Higgs sector. The block has been called NHMIXC and contains the one-loop cor-

\footnotetext{
${ }^{14}$ These are always real.
} 
rected mixing matrix elements $\mathrm{ZH}_{i j}$ in the basis $i=\left(H_{1}, H_{2}, H_{3}, H_{4}, H_{5}\right), j=\left(h_{d}, h_{u}, h_{s}, a, a_{s}\right) 15$. It corresponds to the FORTRAN format

$$
(1 \mathrm{x}, \mathrm{I} 2,1 \mathrm{x}, \mathrm{I} 2,3 \mathrm{x}, 1 \mathrm{P}, \mathrm{E} 16.8,0 \mathrm{P}, 3 \mathrm{x}, \#, 1 \mathrm{x}, \mathrm{A})
$$

so that the structure of the block is as follows,

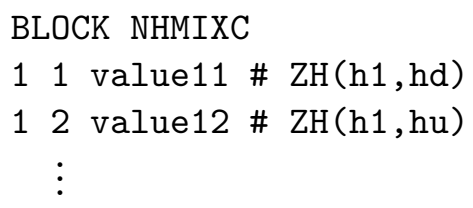

In the SLHA output of the loop corrected complex NMSSM Higgs boson masses, there is some peculiarity. The pdg code for these particles is increased with ascending mass values. This means in particular, that, irrespective of its amount of pseudoscalar admixture, the third heaviest (heaviest) Higgs boson $H_{3}\left(H_{5}\right)$ has the pdg code 36 (46) which in the CP-conserving case is reserved for the pseudoscalar mass eigenstates.

There are several warnings that are issued by the code for the mass calculation. In case one of the obligatory parameters is missing a warning is issued with the name of the missing parameter. If the real NMSSM has been chosen in MODSEL and non-zero imaginary parts are nevertheless filled in IMEXTPAR, the user is warned that these should be zero and the program is terminated. A further warning is issued if a different model than the NMSSM is chosen in the block MODSEL, as the program only calculates the higher order corrections to the NMSSM Higgs boson masses. If the CP-conserving or violating case has not been specified, the user is notified by the program to fill in the information. In case the imaginary parts of $A_{\lambda}$ and $A_{\kappa}$ have been given, the user is warned, that these values are ignored. They are fixed in our renormalisation prescription through the tadpole conditions, and the thus obtained values are given out in the SLHA output to ensure consistency. If both the real part of $A_{\lambda}$ and $M_{H^{ \pm}}$are set in the input file, a warning is issued to use either of these values. Furthermore, the $M_{H^{ \pm}}$value is assumed to be the default input and the value of $A_{\lambda}$ is ignored in this case.

\section{Summary and Outlook}

We have presented the program package NMSSMCALC for the calculation of the loop-corrected NMSSM Higgs boson masses and decay widths in the CP-conserving and the CP-violating NMSSM. The Higgs boson masses are calculated at one-loop order in a mixed renormalisation scheme of onshell and $\overline{\mathrm{DR}}$ conditions. The decays include the most up-to-date higher order corrections. The program will be continuously updated to include the state-of-the art results for Higgs boson masses and decays. In particular we plan to extend the SUSY-QCD corrections to the decays into stops and sbottoms to the complex NMSSM. Furthermore, the two-loop corrections to the Higgs boson masses as well as the higher-order corrections to the trilinear Higgs self-couplings will be included. The program thus serves as a tool for making precise predictions for the masses and the decay widths of the NMSSM Higgs bosons. Therefore it allows to reliably interpret the experimental results and distinguish between the Higgs sectors of models beyond the SM.

\section{Acknowledgments}

J.B., R.G., M.M., D.T.N. and K.W. are supported by the DFG SFB/TR9 "Computational Particle Physics". R.G. acknowledges financial support by the "Landesgraduiertenförderung des Landes

\footnotetext{
${ }^{15}$ Note that $\left(h_{d}, h_{u}, h_{s}, a, a_{s}, G\right)^{T}=\mathcal{R}^{G}\left(h_{d}, h_{u}, h_{s}, a_{d}, a_{u}, a_{s}\right)^{T}$
} 
Baden-Württemberg".

\section{Appendix}

\section{A The input file bhdecay.in}

In the following the various input values of the file bhdecay. in are specified. We have

VTB, VTS, VTD, VCB, VCS, VCD, VUB, VUS, VUD: The CKM matrix elements $V_{t b}, V_{t s}, V_{t d}$, $V_{c b}, V_{c s}, V_{c d}, V_{u b}, V_{u s}$ and $V_{u d}$.

NNLO (M): If $=0$ then the $\mathcal{O}\left(\alpha_{s}\right)$ formula is used for the conversion of the quark pole into the

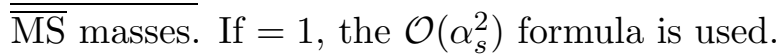

ON-SHELL: If $=0$ then the off-shell decays into $\bar{t} t^{*}, \bar{b} t^{*}, \bar{s} t^{*}, \bar{d} t^{*}$, into a Higgs and off-shell gauge boson and into two off-shell Higgs bosons are calculated. If $=1$, they are not included.

ON-SH-WZ: If $=0$, the double off-shell pair decays into $W^{*} W^{*}, Z^{*} Z^{*}$ are calculated. If $=-1$, the double off-shell decays are included below threshold, but the on-shell decays above. If $=1$, only the single off-shell decays into $W^{*} W, Z^{*} Z$ are calculated below threshold, and the on-shell decays above.

$\underline{\text { OFF-SUSY: }}$ If $=0$, the decays (and loops) into SUSY particles are included. If $=1$, they are excluded.

NF-GG: Number of light flavours $(3,4$ or 5$)$ included in the gluonic decays. 


\section{References}

[1] G. Aad et al. [ATLAS Collaboration], Phys. Lett. B 716 (2012) 1 [arXiv:1207.7214 [hep-ex]]; G. Aad et al. [ATLAS Collaboration], ATLAS-CONF-2012-162.

[2] S. Chatrchyan et al. [CMS Collaboration], Phys. Lett. B 716 (2012) 30 arXiv:1207.7235 [hep-ex]]; S. Chatrchyan et al. [CMS Collaboration], CMS-PAS-HIG-12-045.

[3] J. Goldstone, A. Salam and S. Weinberg, Phys. Rev. 127 (1962) 965; S. Weinberg, Phys. Rev. Lett. 19 (1967) 1264; S.L. Glashow, S. Weinberg, Phys. Rev. Lett. 20 (1968) 224; A. Salam, Proceedings Of The Nobel Symposium, Stockholm 1968, ed. N. Svartholm.

[4] P.W. Higgs, Phys. Lett. 12 (1964) 132; Phys. Rev. Lett. 13 (1964) 508 and Phys. Rev. 145 (1966) 1156; F. Englert and R. Brout, Phys. Rev. Lett. 13 (1964) 321; G.S. Guralnik, C.R. Hagen and T.W. Kibble, Phys. Rev. Lett. 13 (1964) 585.

[5] P. Fayet, Nucl. Phys. B 90 (1975) 104; Phys. Lett. B 64 (1976) 159; Phys. Lett. B 69 (1977) 489 and Phys. Lett. B 84 (1979) 416; H.P. Nilles, M. Srednicki and D. Wyler, Phys. Lett. B 120 (1983) 346; J.M. Frere, D.R. Jones and S. Raby, Nucl. Phys. B 222 (1983) 11; J.P. Derendinger and C.A. Savoy, Nucl. Phys. B 237 (1984) 307; A.I. Veselov, M.I. Vysotsky and K.A. Ter-Martirosian, Sov. Phys. JETP 63 (1986) 489; J.R. Ellis, J.F. Gunion, H.E. Haber, L. Roszkowski and F. Zwirner, Phys. Rev. D 39 (1989) 844; M. Drees, Int. J. Mod. Phys. A 4 (1989) 3635.

[6] U. Ellwanger, M. Rausch de Traubenberg and C.A. Savoy, Phys. Lett. B 315 (1993) 331, Z. Phys. C 67 (1995) 665 and Nucl. Phys. B 492 (1997) 307; U. Ellwanger, Phys. Lett. B 303 (1993) 271; P. Pandita, Z. Phys. C 59 (1993) 575; T. Elliott, S.F. King and P.L. White, Phys. Rev. D 49 (1994) 2435; S.F. King and P.L. White, Phys. Rev. D 52 (1995) 4183; F. Franke and H. Fraas, Int. J. Mod. Phys. A 12 (1997) 479.

[7] For reviews, see: M. Maniatis, Int. J. Mod. Phys. A25 (2010) 3505 arXiv:0906.0777 [hepph]]; U. Ellwanger, C. Hugonie, A. M. Teixeira, Phys. Rept. 496 (2010) 1 arXiv:0910.1785 [hep-ph]]; U. Ellwanger, Eur. Phys. J. C 71 (2011) 1782 [arXiv:1108.0157 [hep-ph]].

[8] M. Bastero-Gil, C. Hugonie, S. F. King, D. P. Roy and S. Vempati, Phys. Lett. B 489, 359 (2000) hep-ph/0006198]; A. Delgado, C. Kolda, J. P. Olson and A. de la Puente, Phys. Rev. Lett. 105, 091802 (2010) arXiv:1005.1282 [hep-ph]]; U. Ellwanger, G. Espitalier-Noel and C. Hugonie, JHEP 1109 (2011) 105 arXiv:1107.2472 [hep-ph]]; G. G. Ross and K. SchmidtHoberg, arXiv:1108.1284 [hep-ph]; S. F. King, M. Muhlleitner and R. Nevzorov, Nucl. Phys. B 860 (2012) 207 arXiv:1201.2671 [hep-ph]]; J. -J. Cao, Z. -X. Heng, J. M. Yang, Y. M. Zhang and J. -Y. Zhu, JHEP 1203 (2012) 086 [arXiv:1202.5821 [hep-ph]]; J. Cao, Z. Heng, J. M. Yang and J. Zhu, JHEP 1210 (2012) 079 arXiv:1207.3698 [hep-ph]]; S. F. King, M. Muhlleitner, R. Nevzorov and K. Walz, Nucl. Phys. B 870 (2013) 323 arXiv:1211.5074 [hep-ph]].

[9] J. Baglio, T. N. Dao, R. Gröber, M. M. Mühlleitner, H. Rzehak, M. Spira, J. Streicher and K. Walz, EPJ Web Conf. 49 (2013) 12001.

[10] K. Ender, T. Graf, M. Mühlleitner and H. Rzehak, Phys. Rev. D 85 (2012) 075024 arXiv:1111.4952 [hep-ph]]. 
[11] T. Graf, R. Gröber, M. Mühlleitner, H. Rzehak and K. Walz, JHEP 1210 (2012) 122 arXiv:1206.6806 [hep-ph]].

[12] U. Ellwanger, Phys. Lett. B303 (1993) 271 hep-ph/9302224; T. Elliott, S. F. King, P. L. White, Phys. Lett. B305 (1993) 71 hep-ph/9302202], Phys. Lett. B314 (1993) 56 hep-ph/9305282], Phys. Rev. D49 (1994) 2435 hep-ph/9308309]; P. N. Pandita, Z. Phys. C59 (1993) 575, Phys. Lett. B318 (1993) 338.

[13] U. Ellwanger, C. Hugonie, Phys. Lett. B623 (2005) 93 hep-ph/0504269].

[14] G. Degrassi and P. Slavich, Nucl. Phys. B 825 (2010) 119 [arXiv:0907.4682 [hep-ph]].

[15] F. Staub, W. Porod, B. Herrmann, JHEP 1010 (2010) 040 [arXiv:1007.4049 [hep-ph]].

[16] S. W. Ham, J. Kim, S. K. Oh and D. Son, Phys. Rev. D 64 (2001) 035007 hep-ph/0104144; S. W. Ham, S. H. Kim, S. K. Oh and D. Son, Phys. Rev. D 76 (2007) 115013 arXiv:0708.2755 [hep-ph]].

[17] S. W. Ham, S. K. Oh and D. Son, Phys. Rev. D 65 (2002) 075004 hep-ph/0110052; S. W. Ham, Y. S. Jeong and S. K. Oh, hep-ph/0308264.

[18] K. Funakubo and S. Tao, Prog. Theor. Phys. 113 (2005) 821 hep-ph/0409294.

[19] K. Cheung, T.--J. Hou, J. S. Lee and E. Senaha, Phys. Rev. D 82 (2010) 075007 arXiv:1006.1458 [hep-ph]].

[20] S. Munir, arXiv:1310.8129 [hep-ph].

[21] D. T. Nhung, M. Mühlleitner, J. Streicher and K. Walz, JHEP 11 (2013) 181 arXiv:1306.3926 [hep-ph]].

[22] A. Djouadi, J. Kalinowski and M. Spira, Comput. Phys. Commun. 108 (1998) 56 hep-ph/9704448]; J. M. Butterworth, F. Maltoni, F. Moortgat, P. Richardson, S. Schumann, P. Skands, J. Alwall and A. Arbey et al., arXiv:1003.1643 [hep-ph]; A. Djouadi, M. M. Mühlleitner and M. Spira, Acta Phys. Polon. B 38 (2007) 635 hep-ph/0609292.

[23] R. Hempfling, Phys. Rev. D49 (1994) 6168; L. Hall, R. Rattazzi and U. Sarid, Phys. Rev. D50 (1994) 7048; M. Carena, M. Olechowski, S. Pokorski and C.E.M. Wagner, Nucl. Phys. B426 (1994) 269; D. M. Pierce, J. A. Bagger, K. T. Matchev and R.--J. Zhang, Nucl. Phys. B 491 (1997) 3 hep-ph/9606211]; M. S. Carena, S. Mrenna and C. E. M. Wagner, Phys. Rev. D 60 (1999) 075010 hep-ph/9808312].

[24] M. S. Carena, D. Garcia, U. Nierste and C. E. M. Wagner, Nucl. Phys. B 577 (2000) 88 hep-ph/9912516]; M. S. Carena, J. R. Ellis, S. Mrenna, A. Pilaftsis and C. E. M. Wagner, Nucl. Phys. B 659 (2003) 145 hep-ph/0211467.

[25] J. Guasch, P. Häfliger and M. Spira, Phys. Rev. D 68 (2003) 115001 hep-ph/0305101.

[26] D. Noth and M. Spira, Phys. Rev. Lett. 101 (2008) 181801 arXiv:0808.0087 [hep-ph]].

[27] D. Noth and M. Spira, JHEP 1106 (2011) 084 [arXiv:1001.1935 [hep-ph]].

[28] L. Mihaila and C. Reisser, JHEP 1008 (2010) 021 [arXiv:1007.0693 [hep-ph]].

[29] P. Z. Skands et al., JHEP 0407 (2004) 036 hep-ph/0311123. 
[30] B. C. Allanach et al., Comput. Phys. Commun. 180 (2009) 8 arXiv:0801.0045 [hep-ph]].

[31] M. Frank, T. Hahn, S. Heinemeyer, W. Hollik, H. Rzehak and G. Weiglein, JHEP 0702 (2007) 047 hep-ph/0611326.

[32] W. Hollik, E. Kraus, M. Roth, C. Rupp, K. Sibold and D. Stockinger, Nucl. Phys. B 639, 3 (2002) hep-ph/0204350.

[33] E. Braaten and J. P. Leveille, Phys. Rev. D 22 (1980) 715; N. Sakai, Phys. Rev. D 22 (1980) 2220; T. Inami and T. Kubota, Nucl. Phys. B 179 (1981) 171; M. Drees and K.-I. Hikasa, Phys. Rev. D 41 (1990) 1547; M. Drees and K.-I. Hikasa, Phys. Lett. B 240 (1990) 455 [Erratum-ibid. B 262 (1991) 497].

[34] S. G. Gorishnii, A. L. Kataev, S. A. Larin and L. R. Surguladze, Mod. Phys. Lett. A 5 (1990) 2703; S. G. Gorishnii, A. L. Kataev, S. A. Larin and L. R. Surguladze, Phys.Rev. D 43 (1991) 1633; A. L. Kataev and V. T. Kim, Mod. Phys. Lett. A 9 (1994) 1309; S. G. Gorishnii, A. L. Kataev and S. A. Larin, Sov. J. Nucl. Phys. 40 (1984) 329 [Yad. Fiz. 40 (1984) 517]; L. R. Surguladze, Phys. Lett. B 341 (1994) 60 hep-ph/9405325; S. A. Larin, T. van Ritbergen and J. A. M. Vermaseren, Phys. Lett. B 362 (1995) 134 [hep-ph/9506465]; K. G. Chetyrkin and A. Kwiatkowski, Nucl. Phys. B 461 (1996) 3 hep-ph/9505358.

[35] K. G. Chetyrkin, Phys. Lett. B 390 (1997) 309 hep-ph/9608318.

[36] P. A. Baikov, K. G. Chetyrkin and J. H. Kühn, Phys. Rev. Lett. 96 (2006) 012003 hep-ph/0511063.

[37] A. Mendez and A. Pomarol, Phys. Lett. B 252 (1990) 461; C.-S. Li and R. J. Oakes, Phys. Rev. D 43 (1991) 855; A. Djouadi and P. Gambino, Phys. Rev. D 51 (1995) 218 [Erratumibid. D 53 (1996) 4111] hep-ph/9406431.

[38] A. Djouadi, J. Kalinowski and P.M. Zerwas, Z. Phys. C 70 (1996) 437; S. Moretti and W.J. Stirling, Phys. Lett. B 347 (1995) 291 and (E) B 366 (1996) 451.

[39] K. G. Chetyrkin, B. A. Kniehl and M. Steinhauser, Nucl. Phys. B 510 (1998) 61 hep-ph/9708255].

[40] M. Krämer, E. Laenen and M. Spira, Nucl. Phys. B 511 (1998) 523 hep-ph/9611272.

[41] Y. Schröder and M. Steinhauser, JHEP 0601 (2006) 051 hep-ph/0512058].

[42] K. G. Chetyrkin, J. H. Kühn and C. Sturm, Nucl. Phys. B 744 (2006) 121 hep-ph/0512060].

[43] T. Inami, T. Kubota and Y. Okada, Z. Phys. C 18 (1983) 69; A. Djouadi, M. Spira and P. M. Zerwas, Phys. Lett. B 264 (1991) 440; K. G. Chetyrkin, B. A. Kniehl and M. Steinhauser, Phys. Rev. Lett. 79 (1997) 353 hep-ph/9705240].

[44] M. Spira, A. Djouadi, D. Graudenz and P. M. Zerwas, Phys. Lett. B 318 (1993) 347.

[45] M. Spira, A. Djouadi, D. Graudenz and P. M. Zerwas, Nucl. Phys. B 453 (1995) 17 hep-ph/9504378.

[46] P. A. Baikov and K. G. Chetyrkin, Phys. Rev. Lett. 97 (2006) 061803 hep-ph/0604194. 
[47] S. Dawson, A. Djouadi and M. Spira, Phys. Rev. Lett. 77 (1996) 16 hep-ph/9603423; A. Djouadi, V. Driesen, W. Hollik and J. I. Illana, Eur. Phys. J. C 1 (1998) 149 hep-ph/9612362.

[48] R. V. Harlander and M. Steinhauser, Phys. Lett. B 574 (2003) 258 hep-ph/0307346]; R. Harlander and M. Steinhauser, Phys. Rev. D 68 (2003) 111701 hep-ph/0308210; R. V. Harlander and F. Hofmann, JHEP 0603 (2006) 050 hep-ph/0507041.

[49] C. Anastasiou, S. Beerli, S. Bucherer, A. Daleo and Z. Kunszt, JHEP 0701 (2007) 082 hep-ph/0611236.

[50] M. Mühlleitner and M. Spira, Nucl. Phys. B 790 (2008) 1 hep-ph/0612254.

[51] C. Anastasiou, S. Beerli and A. Daleo, Phys. Rev. Lett. 100 (2008) 241806 arXiv:0803.3065 [hep-ph]].

[52] G. Degrassi and P. Slavich, Nucl. Phys. B 805 (2008) 267 [arXiv:0806.1495 [hep-ph]].

[53] M. Mühlleitner, H. Rzehak and M. Spira, JHEP 0904 (2009) 023 [arXiv:0812.3815 [hep-ph]].

[54] G. Degrassi and P. Slavich, JHEP 1011 (2010) 044 [arXiv:1007.3465 [hep-ph]]; G. Degrassi, S. Di Vita and P. Slavich, JHEP 1108 (2011) 128 [arXiv:1107.0914 [hep-ph]]; G. Degrassi, S. Di Vita and P. Slavich, Eur. Phys. J. C 72 (2012) 2032 [arXiv:1204.1016 [hep-ph]].

[55] H. Zheng and D. Wu, Phys. Rev. D42 (1990) 3760; A. Djouadi, M. Spira, J. van der Bij and P.M. Zerwas, Phys. Lett. B257 (1991) 187; S. Dawson and R.P. Kauffman, Phys. Rev. D47 (1993) 1264; A. Djouadi, M. Spira and P.M. Zerwas, Phys. Lett. B311 (1993) 255; K. Melnikov and O. Yakovlev, Phys. Lett. B312 (1993) 179; M. Inoue, R. Najima, T. Oka and J. Saito, Mod. Phys. Lett.A9 (1994) 1189.

[56] K. Melnikov, M. Spira and O. I. Yakovlev, Z. Phys. C 64 (1994) 401 hep-ph/9405301].

[57] M. Spira, A. Djouadi and P. M. Zerwas, Phys. Lett. B 276 (1992) 350.

[58] R.N. Cahn, Rep. Prog. Phys. 52 (1989) 389.

[59] For a comprehensive study of the NMSSM Higgs masses, see: D. J. Miller, 2, R. Nevzorov and P. M. Zerwas, Nucl. Phys. B 681 (2004) 3 [hep-ph/0304049].

[60] M. Spira, Fortsch. Phys. 46 (1998) 203 hep-ph/9705337]; A. Djouadi, Phys. Rept. 459 (2008) 1 hep-ph/0503173.

[61] A. Djouadi, J. Kalinowski, P. Ohmann and P. M. Zerwas, Z. Phys. C 74 (1997) 93 hep-ph/9605339.

[62] A. Djouadi, P. Janot, J. Kalinowski and P. M. Zerwas, Phys. Lett. B 376 (1996) 220 hep-ph/9603368.

[63] A. Bartl, H. Eberl, K. Hidaka, T. Kon, W. Majerotto and Y. Yamada, Phys. Lett. B 402 (1997) 303 [hep-ph/9701398]; A. Arhrib, A. Djouadi, W. Hollik and C. Junger, Phys. Rev. D 57 (1998) 5860 [hep-ph/9702426].

[64] H. Eberl, K. Hidaka, S. Kraml, W. Majerotto and Y. Yamada, Phys. Rev. D 62 (2000) 055006 hep-ph/9912463. 
[65] E. Accomando, G. Chachamis, F. Fugel, M. Spira and M. Walser, Phys. Rev. D 85 (2012) 015004 arXiv:1103.4283 [hep-ph]].

[66] J. Ellis, M.K. Gaillard and D.V. Nanopoulos, Nucl. Phys. B106 (1976) 292; H. Georgi, S. Glashow, M. Machacek and D.V. Nanopoulos, Phys. Rev. Lett. 40 (1978) 692.

[67] A.I. Vainshtein, M.B. Voloshin, V.I. Zakharov and M.A. Shifman, Sov. J. Nucl. Phys. 30 (1979) 711.

[68] J.A. Coarasa, R.A. Jiménez and J. Solà, Phys. Lett. B 389 (1996) 312.

[69] R. Contino, M. Ghezzi, C. Grojean, M. Mühlleitner and M. Spira, JHEP 1307 (2013) 035 arXiv:1303.3876 [hep-ph]]. 